\title{
Characteristics of Joint Involvement and Relationships with Systemic Inflammation in Systemic Sclerosis: Results from the EULAR Scleroderma Trial and Research Group (EUSTAR) Database
}

\author{
JEROME AVOUAC, ULRICH WALKER, ALAN TYNDALL, ANDRÉ KAHAN, MARCO MATUCCI-CERINIC, \\ YANNICK ALLANORE, and EUSTAR
}

\begin{abstract}
Objective. To determine the prevalence of and independent factors associated with joint involvement in a large population of patients with systemic sclerosis $(\mathrm{SSc})$.

Methods. This study was cross-sectional, based on data collected on patients included in the European League Against Rheumatism (EULAR) Scleroderma Trials and Research (EUSTAR) registry. We queried this database to extract data regarding global evaluation of patients with SSc and the presence of any clinical articular involvement: synovitis (tender and swollen joints), tendon friction rubs (rubbing sensation detected as the tendon was moved), and joint contracture (stiffness of the joints that decreased their range of motion). Overall joint involvement was defined by the occurrence of synovitis and/or joint contracture and/or tendon friction rubs.

Results. We recruited 7286 patients with SSc; their mean age was $56 \pm 14$ years, disease duration 10 \pm 9 years, and $4210(58 \%)$ had a limited cutaneous disease subset. Frequencies of synovitis, tendon friction rubs, and joint contractures were $16 \%, 11 \%$, and $31 \%$, respectively. Synovitis, tendon friction rubs, and joint contracture were more prevalent in patients with the diffuse cutaneous subset and were associated together and with severe vascular, muscular, renal, and interstitial lung involvement. Moreover, synovitis had the highest strength of association with elevated acute-phase reactants taken as the dependent variable.

Conclusion. Our results highlight the striking level of articular involvement in SSc, as evaluated by systematic examination in a large cohort of patients with SSc. Our data also show that synovitis, joint contracture, and tendon friction rubs are associated with a more severe disease and with systemic inflammation. (First Release June 15 2010; J Rheumatol 2010;37:1488-501; doi:10.3899/jrheum.091165)
\end{abstract}

Key Indexing Terms:

SYSTEMIC SCLEROSIS

JOINT CONTRACTURE

JOINT INVOLVEMENT SYNOVITIS

TENDON FRICTION RUB

Systemic sclerosis ( $\mathrm{SSc}$ ) is a severe connective tissue disease characterized by vascular, immune, and fibrotic changes in the skin and some internal organs ${ }^{1}$. SSc is incur-

From the Université Paris Descartes, AP-HP, Hôpital Cochin, Service de Rhumatologie A, Paris, France; Rheumatologische Universitätsklinik Felix Platter Spital, Basel, Switzerland; and the Department of BioMedicine, Division of Rheumatology AOUC, University of Florence, Florence, Italy.

EUSTAR is supported by a research grant from EULAR and is under the auspices of the Standing Committee for International Studies Including Clinical Trials (ESCCA).

J. Avouac, MD, PhD; A. Kahan, MD; Y. Allanore, MD, PhD, Université Paris Descartes, AP-HP, Hôpital Cochin, Service de Rhumatologie; U.A. Walker, MD; A. Tyndall, MD, Rheumatologische Universitätsklinik Felix Platter Spital; M. Matucci-Cerinic, MD, PhD, Department of BioMedicine, Division of Rheumatology AOUC, University of Florence.

Address correspondence to Dr. Y. Allanore, Service de Rhumatologie A, Hôpital Cochin, Université Paris Descartes, 27 rue du Faubourg

Saint-Jacques, 75014 Paris, France.

E-mail: yannick.allanore@cch.aphp.fr

Accepted for publication February 23, 2010. able and can influence all aspects of an individual's life, including the performance of everyday occupations ${ }^{2}$. Impaired hand function, characterized by decreased hand mobility, reduced dexterity, and decreased grip force, has been clearly identified by patients with SSc as a major source of difficulty in their activities of daily living ${ }^{3}$. Joint involvement has been shown to strongly contribute to impaired hand function in SSc, leading to disability and impaired quality of life and highlighting the importance of joint involvement in $\mathrm{SSc}^{4-8}$. Studies have shown the striking level of radiological hand involvement at the articular, bone,

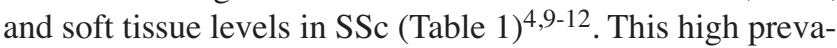
lence of hand and wrist joint pathology was confirmed by ultrasonography in a recent study performed with 45 patients with SSc (Table 1) ${ }^{13}$. Moreover, the usefulness of magnetic resonance imaging (MRI) was additionally emphasized in the accurate diagnosis and characterization of SSc-associated hand arthropathy (Table 1) ${ }^{14,15}$. Although these studies ought to increase the understanding of osteo-

Personal non-commercial use only. The Journal of Rheumatology Copyright (C) 2010. All rights reserved. 
Table 1. Hand involvement in systemic sclerosis, assessed by radiographs, ultrasonography, and magnetic resonance imaging (MRI).

\begin{tabular}{|c|c|c|c|c|c|c|}
\hline \multirow{2}{*}{$\begin{array}{l}\text { Hand Condition } \\
\text { and Joint }\end{array}$} & \multicolumn{4}{|c|}{ Radiography } & \multirow{2}{*}{$\begin{array}{l}\text { Ultrasonography } \\
\begin{array}{c}\text { Cuomo } \\
\text { n }\end{array} \\
\text { n }\end{array}$} & \multirow{2}{*}{$\begin{array}{c}\text { MRI } \\
\text { Low }^{14} \\
\mathrm{n}=17\end{array}$} \\
\hline & $\begin{array}{c}\text { Avouac }{ }^{9}, \\
\mathrm{n}=120\end{array}$ & $\begin{array}{c}\text { Baron }^{4}, \\
\mathrm{n}=38\end{array}$ & $\begin{array}{l}\text { La Montagna }^{10} \\
\mathrm{n}=76\end{array}$ & $\begin{array}{c}\text { Brun }^{11}, \\
\mathrm{n}=41\end{array}$ & & \\
\hline \multicolumn{7}{|l|}{ Joint involvement, n (\%) } \\
\hline Erosion & $25(21)$ & $15(40)$ & $8(10.5)$ & ND & $5(11)$ & $7(41)$ \\
\hline Wrist & 17 & 4 & ND & ND & 1 & 2 \\
\hline $\mathrm{MCP}$ & 9 & 9 & ND & ND & 4 & 5 \\
\hline $\mathrm{PIP}$ & 10 & 3 & ND & ND & 0 & 2 \\
\hline DIP & 18 & 7 & ND & ND & 0 & 0 \\
\hline Joint space narrowing, $\mathrm{n}(\%)$ & $35(28)$ & $13(34)$ & ND & $10(24)$ & $8(18)$ & ND \\
\hline Wrist & 13 & 2 & $13(17)$ & ND & 0 & ND \\
\hline $\mathrm{MCP}$ & 12 & 2 & ND & ND & 8 & ND \\
\hline PIP & 14 & 4 & $31(41)$ & ND & 2 & ND \\
\hline DIP & 25 & 12 & $41(54)$ & ND & 0 & ND \\
\hline Synovitis, n (\%) & NA & NA & NA & NA & $22(49)$ & $8(47)$ \\
\hline Wrist & & & & & 2 & 2 \\
\hline $\mathrm{MCP}$ & & & & & 15 & 5 \\
\hline PIP & & & & & 12 & 1 \\
\hline DIP & & & & & 1 & 0 \\
\hline Synovial proliferation, $\mathrm{n}(\%)$ & NA & NA & NA & NA & $19(42)$ & ND \\
\hline Wrist & & & & & 2 & $\mathrm{ND}$ \\
\hline $\mathrm{MCP}$ & & & & & 12 & ND \\
\hline PIP & & & & & 2 & ND \\
\hline DIP & & & & & 0 & ND \\
\hline Tenosynovitis, n (\%) & NA & NA & NA & NA & ND & $8(47)$ \\
\hline Flexor & & & & & ND & 7 \\
\hline Extensor & & & & & ND & 3 \\
\hline \multicolumn{7}{|l|}{ Bone involvement, $\mathrm{n}(\%)$} \\
\hline Radiological demineralization & $28(23)$ & $16(42)$ & $12(16)$ & $7(17)$ & ND & $\mathrm{ND}$ \\
\hline Acroosteolysis & $26(22)$ & $14(37)$ & $22(29)$ & $11(27)$ & ND & ND \\
\hline Bone edema & NA & NA & NA & NA & NA & $9(53)$ \\
\hline \multicolumn{7}{|l|}{ Soft tissue involvement, $\mathrm{n}(\%)$} \\
\hline Flexion contracture & $32(27)$ & ND & $31(41)$ & ND & NA & ND \\
\hline Calcinosis & $28(23)$ & $19(50)$ & $45(59)$ & $18(44)$ & $12(27)$ & ND \\
\hline
\end{tabular}

ND: no data; NA: not applicable; MRI: magnetic resonance imaging; MCP: metacarpophalangeal joint; PIP: proximal interphalangeal joint; DIP: distal interphalangeal joint.

articular involvement, few data are available on the prevalence of clinical joint involvement, which has not yet been defined accurately. At some time in the disease course, patients with SSc may develop joint involvement. This manifests clinically as arthralgia, arthritis, joint contracture, and/or tendon sheath involvement. Joint involvement may even predate the development of classical features of the disease ${ }^{16,17}$. Joint symptoms have been noted in different series in $12 \%$ to $66 \%$ of patients at the time of diagnosis and in $24 \%$ to $97 \%$ of patients at some time during the course of their illness ${ }^{4,16}$. Histological evidence of inflammation with lymphocytic and plasma-cell infiltration has been found in up to $66 \%$ of synovial biopsies from patients with $\mathrm{SSc}^{4}$.

This wide variation, and disparities in the reported prevalence of clinical features, as well as the scarce data available about their association with other disease measurements/phenotypes, requires clarification in a large population of patients with SSc. We aimed to determine the point prevalence of joint involvement (synovitis, joint contrac- ture, and tendon friction rubs) in a large population of Europeans with SSc and to identify disease-phenotype associations.

\section{MATERIALS AND METHODS}

The European League Against Rheumatism (EULAR) Scleroderma Trials and Research (EUSTAR) Joint Study was cross-sectional, based on data collected on patients with SSc who were included in the EUSTAR registry. This database was launched in June 2004 and documents a multinational, prospective, and open SSc cohort. Since 2004, 150 participating medical centers entered consecutive patients into a registry and all data into a specific database, which was locked for this study in April 2008. The structure and Minimal Essential Dataset (MEDS) of the EUSTAR database have been described ${ }^{18-20}$. The MEDS was constructed in consensus by the EUSTAR members, and covers demographic aspects, disease duration, organ involvement, and laboratory data. Baseline data collected during the first patient visit to a EUSTAR center were analyzed for the purpose of our study. All patients included in either database granted their informed consent to participate, and appropriate institutional ethics committees approved the research program.

The data regarding the presence of articular involvement was assessed as "yes" or "no" in the following manner: (1) synovitis (defined by tender 
and swollen joints); (2) tendon friction rubs (defined by a leathery, rubbing, "squeaking" sensation detected as the tendon was moved actively or passively); and (3) joint contracture (defined by stiffness of the joint that decreased range of motion and prevented full extension). Overall joint involvement was defined, for the purpose of our study, by the occurrence of synovitis and/or joint contracture and/or tendon friction rubs in at least 1 area or 1 joint.

Global evaluation of patients with SSc was also assessed and extracted from the MEDS data (Figure 1) ${ }^{18}$. Global evaluation was based on the collection of clinical variables, including distinction of the cutaneous subset of the disease according to the LeRoy criteria ${ }^{21}$, disease duration (date of first non-Raynaud symptom), and presence of active or past digital ulcerations. Pulmonary involvement was recorded as present if pulmonary fibrosis was seen on plain chest radiographs and/or by the presence of abnormal respiratory function tests (carbon monoxide diffusion capacity). Pulmonary hypertension was recorded as present by the finding of systolic pulmonary artery pressure (SPAP) $>40 \mathrm{~mm} \mathrm{Hg}$ on an echocardiogram. Renal involvement was recorded as present if there was a history of hypertensive renal crisis or by the presence of proteinuria (+ or more on a urinalysis dipstick). Muscle involvement was recorded as present if muscle weakness and/or elevated creatine phosphokinase (CPK) was present. Inflammatory markers were recorded as present if the erythrocyte sedimentation rate (ESR) was $>$ $28 \mathrm{~mm} / \mathrm{h}$ or by $\mathrm{C}$-reactive protein $>10 \mathrm{mg} / \mathrm{l}$. The following serological tests were recorded as present or absent: antinuclear antibodies (ANA), anticentromere antibodies (by immunofluorescence on HEp-2 cells), and antitopoisomerase I antibodies (counter immunoelectrophoresis and/or immunodiffusion).

Statistical analysis. All data are presented as mean (SD) for continuous variables and numbers (percentages) for categorical variables, unless stated otherwise. Data were statistically analyzed using chi-square tests for differences in frequency and the Student's t-test for comparison between 2 normally distributed continuous variables. We applied a Bonferroni correction for multiple comparisons. To this end, we divided 0.05 by the number of disease characteristics tested (17 sets of variables) and obtained a corrected probability value of 0.003 . Thus, probability value $\leq 0.003$ was considered statistically significant. A multivariate stepwise logistic regression

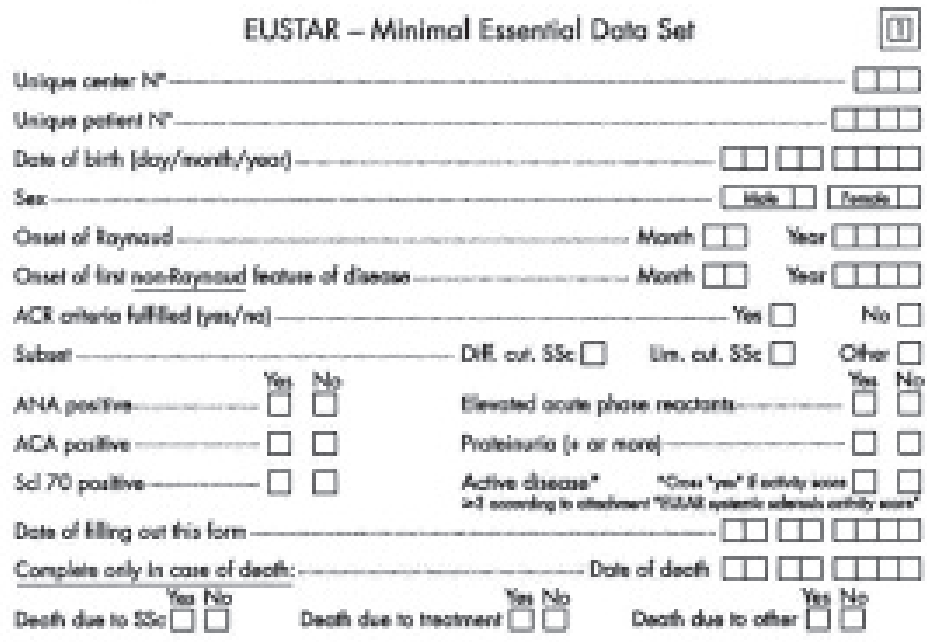

EUSTAR - Minimal Essential Dala Set

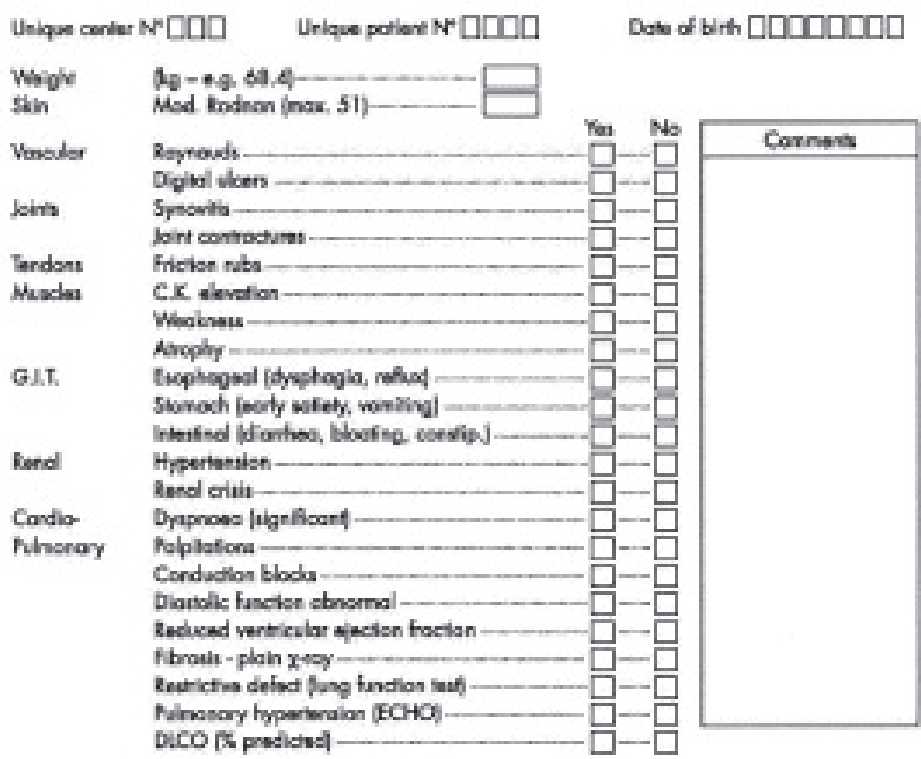

Figure 1. Items of the Minimal Essential Dataset (MEDS). From Walker, et al. Ann Rheum Dis 2007;68:754-6318; with permission. 
analysis was also performed for all variables identified with $\mathrm{p} \leq 0.10$ univariately, with calculation of OR estimates and 95\% CI (OR and probability value were not provided by the software when not significant $)^{19}$. The OR is a measure of effect size, used as a descriptive statistic in logistic regression analysis, describing the strength of association between 2 data values. Unlike other measures of association for paired data such as the relative risk, the OR treats the 2 variables being compared symmetrically, and can be estimated using some types of nonrandom samples. In this model, probability value $<0.05$ was considered statistically significant.

\section{RESULTS}

Study population. We included 7286 successive patients with SSc, of whom $6266(86 \%)$ were women. The mean age of the patients was $56 \pm 14$ years and the mean disease duration was $10 \pm 9$ years; 4210 had the limited cutaneous subtype, 2393 the diffuse cutaneous subtype, and it was not possible to classify 683 patients according to the LeRoy criteria ${ }^{21}$. The other patient characteristics are provided in Table 2.

Prevalence of articular manifestations. The point prevalence of synovitis was $16 \%(1191 / 7286)$. Tendon friction rubs and joint contracture were found in the whole SSc population in $802 / 7286(11 \%)$ and 2264/7286 (31\%) patients, respectively (Figure $2 \mathrm{~A}$ ). The number of patients with any of these 3 features, defining overall joint involvement, was 2025/7286 (28\%).

Association of articular involvement with other subphenotypes. The detailed results of association between some articular involvement (respectively, synovitis, joint contracture, and tendon friction rubs) and other subsets of patients with SSc are provided in Tables 3 to 8 .

Table 2. Characteristics of patients presenting with systemic sclerosis who were included in the EUSTAR database up to April 2008.

\begin{tabular}{lc}
\hline Characteristic & Patients with SSc, \\
& $\mathrm{n}=7286$ \\
\hline Age, mean \pm SD, yrs & $56 \pm 14$ \\
Men/women, $\mathrm{n}(\%)$ & $1020(14) / 6266(86)$ \\
Disease duration, mean \pm SD, yrs & $10 \pm 9$ \\
Cutaneous subtype, $\mathrm{n}(\%)$ & \\
$\quad$ Limited & $4210(58)$ \\
$\quad$ Diffuse & $2393(33)$ \\
$\quad$ Not classified & $683(9)$ \\
Raynaud phenomenon, $\mathrm{n}(\%)$ & $6946(95)$ \\
Digital ulceration, $\mathrm{n}(\%)$ & $2291(31)$ \\
Muscle weakness, $\mathrm{n}(\%)$ & $1950(27)$ \\
Pulmonary fibrosis, $\mathrm{n}(\%)$ & $2607(36)$ \\
Elevated systolic pulmonary artery pressure, $\mathrm{n}(\%)$ & $1756(24)$ \\
Renal crisis, $\mathrm{n}(\%)$ & $160(2)$ \\
Positive antinuclear antibodies, $\mathrm{n}(\%)$ & $6617(91)$ \\
Positive antitopoisomerase-1 antibodies, $\mathrm{n}(\%)$ & $2293(31)$ \\
Positive anticentromere antibodies, $\mathrm{n}(\%)$ & $2396(33)$ \\
Elevated creatine phosphokinase $(\mathrm{CPK}), \mathrm{n}(\%)$ & $581(8)$ \\
Elevation of acute-phase reactants, $\mathrm{n}(\%)$ & $2153(29)$ \\
Proteinuria, $\mathrm{n}(\%)$ & $435(6)$ \\
Active disease according to European score, $\mathrm{n}(\%)$ & $2118(29)$ \\
\hline
\end{tabular}

EUSTAR: European League Against Rheumatism Scleroderma Trials and Research; SSc: systemic sclerosis.
The frequency of synovitis was significantly higher in patients with the diffuse cutaneous subset compared to the limited cutaneous subtype (484/2393, 20\%, vs 570/4210, $13.5 \%$; $<<0.05$; Figure $2 \mathrm{~B})$. In the whole population of patients with SSc, using multivariate stepwise logistic regression, synovitis was associated with markers of severe vascular (elevated SPAP > $40 \mathrm{~mm} \mathrm{Hg}$ ) and muscular (muscle weakness) involvement (Table 3 ). The presence of synovitis was also associated with elevated acute-phase reactants (Table 3). It is noteworthy that, in another multivariate model with elevated acute-phase reactants taken as the dependent variable, synovitis had the highest strength of association, with an OR of 2.10, 95\% CI 1.67-2.64.

Subgroup analyses according to the cutaneous subset showed that patients with synovitis and either limited or diffuse cutaneous SSc were more likely to experience severe disease and systemic inflammation (Table 3).

Synovitis was present in patients with SSc in all disease stages: indeed, 460/1191 (39\%) patients with SSc with synovitis had disease duration < 5 years, 345/2196 (29\%) had disease duration between 5 and 10 years, and 386/2669 $(32 \%)$ had disease duration $>10$ years. Subgroup analyses according to disease duration also showed that the likelihood of the diffuse cutaneous subset was significantly higher only in the subgroup of patients with synovitis and early disease (date of first non-Raynaud symptom, $<5$ years). Moreover, associations identified in the whole SSc population, between synovitis and criteria of severe disease and systemic inflammation, were found in all disease stages (Table 4).

As expected, the prevalence of tendon friction rubs was significantly higher in patients with the diffuse cutaneous subset (486/2393, 20\%, vs 260/4210, 6\%; p < 0.05; Figure $2 \mathrm{~B})$. In the whole population of patients with $\mathrm{SSc}$, this symptom indicated the existence of a severe vascular, interstitial lung, and renal involvement (Table 5). In patients with the diffuse cutaneous involvement, tendon friction rubs were noted most often in younger patients and early in the disease. The subset of patients with tendon friction rubs and diffuse cutaneous SSc was more likely to experience severe muscular, vascular, and kidney involvement. Patients with limited skin thickening who had friction rubs also experienced a more severe disease (digital ulcers, pulmonary fibrosis, muscle weakness) and had higher frequency of antitopoisomerase-1 antibodies (37\% vs 19\%), which may suggest that these patients may represent a subset of individuals with "subclinical" or "aborted" diffuse SSc (Table 5). Altogether this suggests that tendon friction rubs can be regarded as a marker of severity of SSc.

Tendon friction was noted in all disease stages, but tended to occur more often in early disease: 322/802 (40\%) patients with SSc with tendon friction rubs had a disease duration $<5$ years, 213/802 (27\%) between 5 and 10 years, and $267 / 802(33 \%)>10$ years. Sensitivity analyses accord-

Personal non-commercial use only. The Journal of Rheumatology Copyright (C) 2010. All rights reserved. 


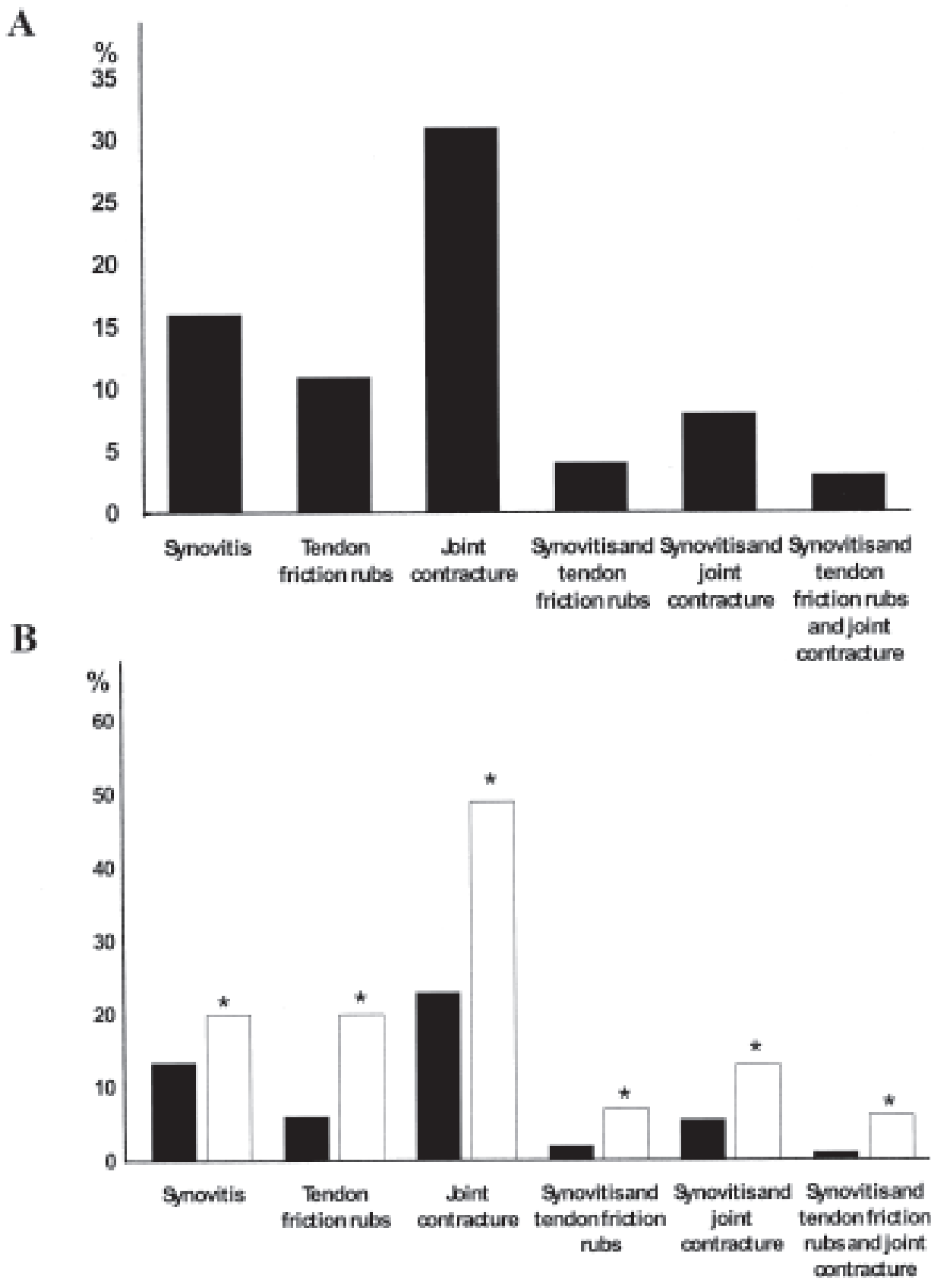

- Limited cutaneous subtype $\quad \square$ Diffuse cutaneous subkype

Figure 2. A. Prevalence of joint involvement in the whole population of patients with SSc. B. Prevalence of joint involvement in populations with the diffuse and limited cutaneous disease subsets. *Diffuse versus limited cutaneous subsets, $\mathrm{p}<0.05$.

ing to disease duration revealed that the likelihood of tendon friction rubs was higher in the patients with diffuse cutaneous SSc in all disease stages, but with a higher OR in the subset of patients with early disease (OR 2.58 in patients with disease duration $<5$ years vs OR 1.54 and 2.09 , respectively, for patients with disease duration between 5 and 10 years and $>10$ years; Table 6). Moreover, patients with early friction rubs were more likely to experience severe disease, regardless of the disease stage (Table 6).

Like synovitis and tendon friction rubs, the prevalence of joint contracture was significantly higher in the diffuse cutaneous subtype $(1167 / 2393$, 49\%, vs $975 / 4210,23 \%$; p < 0.05 ; Figure $2 \mathrm{~B}$ ). In the whole SSc population, joint contracture was associated with severe vascular and interstitial

Personal non-commercial use only. The Journal of Rheumatology Copyright (C) 2010. All rights reserved. 
Table 3. Disease phenotype associations in patients with SSc with or without synovitis.

\begin{tabular}{|c|c|c|c|c|c|c|c|c|c|c|c|c|}
\hline \multirow[t]{2}{*}{$\begin{array}{l}\text { Patient } \\
\text { Characteristic }\end{array}$} & \multicolumn{4}{|c|}{$\begin{array}{l}\text { Whole SSc Population, } \\
\qquad \mathrm{n}=7286\end{array}$} & \multicolumn{4}{|c|}{$\begin{array}{l}\text { Patients with Diffuse Cutaneous } \\
\text { Subtype, } \mathrm{n}=2393\end{array}$} & \multicolumn{4}{|c|}{$\begin{array}{c}\text { Patients with Limited } \\
\text { Cutaneous Subtype, } n=4210\end{array}$} \\
\hline & $\begin{array}{c}\text { With } \\
\text { Synovitis, } \\
\mathrm{n}=1191\end{array}$ & $\begin{array}{c}\text { Without } \\
\text { Synovitis, } \\
\mathrm{n}=6095\end{array}$ & $\mathrm{p}$ & $\begin{array}{c}\text { Stepwise } \\
\text { Regression, } \\
\text { OR } \\
(95 \% \mathrm{CI})\end{array}$ & $\begin{array}{c}\text { With } \\
\text { Synovitis, } \\
\mathrm{n}=484\end{array}$ & $\begin{array}{c}\text { Without } \\
\text { Synovitis, } \\
\mathrm{n}=1909\end{array}$ & $\mathrm{p}$ & $\begin{array}{c}\text { Stepwise } \\
\text { Regression, } \\
\text { OR } \\
(95 \% \mathrm{CI})\end{array}$ & $\begin{array}{c}\text { With } \\
\text { Synovitis, } \\
\mathrm{n}=570\end{array}$ & $\begin{array}{c}\text { Without } \\
\text { Synovitis, } \\
\mathrm{n}=3640\end{array}$ & $\mathrm{p}$ & $\begin{array}{c}\text { Stepwise } \\
\text { Regression } \\
\text { OR } \\
(95 \% \mathrm{CI})\end{array}$ \\
\hline Age, mean $\pm \mathrm{SD}$, yrs & $58 \pm 16$ & $58 \pm 15$ & 0.8 & NS & $54.7 \pm 14$ & $55.5 \pm 15$ & 0.3 & NS & $60.4 \pm 16$ & $60.0 \pm 17$ & 0.6 & NS \\
\hline Friction rub, n (\%) & $291(24)$ & $511(8)$ & $<0.0001^{*}$ & $\begin{array}{c}2.21 \\
(1.82-2.67)\end{array}$ & $173(36)$ & $313(16)$ & $<0.0001^{*}$ & $\begin{array}{c}2.07 \\
(1.62-2.65)\end{array}$ & $93(16)$ & $167(5)$ & $<0.0001^{*}$ & $\begin{array}{c}2.67 \\
(1.96-3.63)\end{array}$ \\
\hline Joint contracture, $\mathrm{n}(\%)$ & $587(49)$ & $1677(27)$ & $<0.0001^{*}$ & $\begin{array}{c}1.81 \\
(1.55-2.11)\end{array}$ & $302(62)$ & $865(45)$ & $<0.0001^{*}$ & $\begin{array}{c}1.38 \\
(1.09-1.74)\end{array}$ & $240(42)$ & $735(20)$ & $<0.0001 *$ & $\begin{array}{c}2.12 \\
(1.72-2.62)\end{array}$ \\
\hline $\begin{array}{l}\text { Raynaud phenomenon, } \\
\mathrm{n}(\%)\end{array}$ & $1144(96)$ & $5802(95)$ & 0.36 & NS & $466(96)$ & $1822(95)$ & 0.7 & NS & $551(97)$ & $3488(96)$ & 0.5 & NS \\
\hline Pulmonary fibrosis, n (\%) & $505(42)$ & $2102(34)$ & $<0.0001 *$ & NS & 266 & 951 & $0.09^{*}$ & NS & 193 & $1023(28)$ & $<0.0001^{*}$ & NS \\
\hline $\begin{array}{l}\text { Elevated systolic } \\
\text { pulmonary artery } \\
\text { pressure, n }(\%)\end{array}$ & $408(34)$ & $1348(22)$ & $<0.0001^{*}$ & $\begin{array}{c}1.49 \\
(1.28-1.73)\end{array}$ & $185(38)$ & $453(24)$ & $<0.0001^{*}$ & $\begin{array}{c}1.51 \\
(1.20-1.91)\end{array}$ & $182(32)$ & $770(21)$ & $<0.0001^{*}$ & $\begin{array}{c}1.52 \\
(1.22-1.89)\end{array}$ \\
\hline Renal crisis, $\mathrm{n}(\%)$ & $41(3.5)$ & $119(2)$ & $0.002 *$ & NS & $29(6)$ & $72(4)$ & $0.04 *$ & NS & $8(1)$ & $41(1)$ & 0.6 & NS \\
\hline $\begin{array}{l}\text { Positive antinuclear } \\
\text { antibodies, } \mathrm{n}(\%)\end{array}$ & $1094(92)$ & $5523(91)$ & 0.97 & NS & 437 (90) & $1734(91)$ & 0.9 & NS & $516(91)$ & 3347 (92) & 0.3 & NS \\
\hline $\begin{array}{l}\text { Positive } \\
\text { antitopoisomerase-1 } \\
\text { antibodies, n (\%) }\end{array}$ & 497 (42) & $1796(29)$ & $<0.0001 *$ & $\begin{array}{c}1.29 \\
(1.08-1.53)\end{array}$ & $319(66)$ & 998 (52) & $<0.0001 *$ & NS & $144(25)$ & 714 (20) & $0.002 *$ & NS \\
\hline $\begin{array}{l}\text { Positive anticentromere } \\
\text { antibodies, } \mathrm{n}(\%)\end{array}$ & $286(24)$ & $2110(35)$ & $<0.0001^{*}$ & NS & $29(6)$ & $111(6)$ & 0.9 & NS & $230(40)$ & $1821(50)$ & $<0.0001^{*}$ & NS \\
\hline
\end{tabular}

NS: not significant; CPK: creatine phosphokinase; SSc: systemic sclerosis. * Variables included for the multivariate stepwise logistic regression analysis.

lung involvement (Table 7). Patients with joint contracture and either diffuse or limited cutaneous SSc were more likely to experience severe vascular and muscular disease, as well as to have elevated acute-phase reactants.

Joint contracture was found in patients with SSc at all disease stages: 745/2264 (33\%) patients with joint contracture had disease duration $<5$ years, 538/2264 (24\%) had disease duration between 5 and 10 years, and 981/2264 (43\%) had disease duration $>10$ years. The likelihoods of diffuse cutaneous subset and criteria for severe vascular, muscular, and interstitial disease were significantly higher in patients with joint contracture, regardless of disease duration (Table 8).

Overall joint involvement (defined by the presence of synovitis and/or joint contracture and/or tendon friction rubs) was significantly more prevalent in multivariate analyses in patients with the diffuse cutaneous subtype (1005/2393 patients, $42 \%$, vs $1020 / 4210$ patients, $24 \%$; $\mathrm{p}<$ $0.05)$. It was associated with markers of severe vascular (digital ulcers with OR 1.80, 95\% CI 1.60-2.03; and elevated SPAP > $40 \mathrm{~mm} \mathrm{Hg}$ with OR 1.62, 95\% CI 1.42-1.84), muscular (muscle weakness with OR 1.65, 95\% CI 1.45-1.87), and interstitial lung involvement (pulmonary fibrosis with OR 1.14, 95\% CI 1.01-1.28). Joint involvement also reflected disease activity and it was associated with elevated acute-phase reactants (OR 1.66, 95\% CI 1.46-1.88).

It is noteworthy that these symptoms were more likely to occur together. Synovitis, taken as the dependent variable in our multivariate model, was associated with the presence of joint contracture (OR 1.81, 95\% CI 1.55-2.11) and tendon friction rubs (OR 2.21, 95\% CI 1.82-2.67). Tendon friction rubs were associated with synovitis (OR 2.31, 95\% CI 1.91-2.71) and joint contracture (OR 3.04, 95\% CI 2.54-3.65), respectively, and joint contracture was associated with the presence of synovitis (OR 1.75, 95\% CI 1.49-2.05) and tendon friction rubs (OR 2.89, 95\% CI 2.39-3.50), respectively (Tables 3, 5, and 7). These associations remained significant in the different subgroup analyses, within cutaneous subsets and disease duration (Tables 3 to 8 ).

Personal non-commercial use only. The Journal of Rheumatology Copyright @ C 2010. All rights reserved. 
Table 4. Disease characteristics associated with synovitis according to disease duration.

\begin{tabular}{|c|c|c|c|c|c|c|c|c|c|c|c|c|}
\hline \multirow[t]{2}{*}{ Characteristic } & \multicolumn{4}{|c|}{$\begin{array}{l}\text { Patients with Disease Duration, } \\
<5 \text { Years, } \mathrm{n}=2421\end{array}$} & \multicolumn{4}{|c|}{$\begin{array}{c}\text { Patients with Disease Duration } \\
\text { Between } 5 \text { and } 10 \text { Years, } \mathrm{n}=2196\end{array}$} & \multicolumn{4}{|c|}{$\begin{array}{l}\text { Patients with Disease Duration } \\
\qquad>10 \text { Years, } n=2669\end{array}$} \\
\hline & $\begin{array}{c}\text { With } \\
\text { Synovitis, } \\
n=460\end{array}$ & $\begin{array}{c}\text { Without } \\
\text { Synovitis, } \\
\text { n=1961 }\end{array}$ & $\mathrm{p}$ & $\begin{array}{c}\text { Stepwise } \\
\text { Regression, } \\
\text { OR } \\
(95 \% \mathrm{CI})\end{array}$ & $\begin{array}{c}\text { With } \\
\text { Synovitis, } \\
\mathrm{n}=345\end{array}$ & $\begin{array}{c}\text { Without } \\
\text { Synovitis, } \\
\mathrm{n}=1851\end{array}$ & $\mathrm{p}$ & $\begin{array}{c}\text { Stepwise } \\
\text { Regression, } \\
\text { OR } \\
(95 \% \mathrm{CI})\end{array}$ & $\begin{array}{c}\text { With } \\
\text { Synovitis, } \\
\mathrm{n}=386\end{array}$ & $\begin{array}{c}\text { Without } \\
\text { Synovitis, } \\
\mathrm{n}=2283\end{array}$ & $\mathrm{p}$ & $\begin{array}{c}\text { Stepwise } \\
\text { Regression } \\
\text { OR } \\
(95 \% \mathrm{CI})\end{array}$ \\
\hline Age, mean $\pm \mathrm{SD}$, yrs & $55.5 \pm 11$ & $56.2 \pm 12$ & 0.4 & NS & $56.3 \pm 14$ & $56.8 \pm 12$ & 0.6 & NS & $60.9 \pm 15$ & $61.3 \pm 16$ & 0.5 & NS \\
\hline Females (\%) & $368(80)$ & $1582(81)$ & 0.5 & NS & $306(89)$ & $1579(85)$ & 0.3 & NS & $355(92)$ & $2076(91)$ & 0.8 & NS \\
\hline $\begin{array}{l}\text { Diffuse cutaneous, } \\
\text { subtype, n }(\%)\end{array}$ & $233(51)$ & $784(39)$ & $<0.0001 *$ & $\begin{array}{c}1.96 \\
(1.45-2.51)\end{array}$ & $114(33)$ & $521(28)$ & $0.002 *$ & NS & $137(35)$ & $604(26)$ & $0.001 *$ & NS \\
\hline Friction rub, n (\%) & $121(26)$ & $201(10)$ & $<0.0001 *$ & $\begin{array}{c}2.51 \\
(1.87-3.39)\end{array}$ & $66(19)$ & $147(8)$ & $<0.0001 *$ & $\begin{array}{c}1.82 \\
(1.33-2.42)\end{array}$ & $104(27)$ & $163(7)$ & $<0.0001^{*}$ & $\begin{array}{c}2.84 \\
(2.05-3.93)\end{array}$ \\
\hline Joint contracture, n (\%) & $214(46)$ & $531(27)$ & $<0.0001^{*}$ & $\begin{array}{c}1.75 \\
(1.37-2.24)\end{array}$ & $147(43)$ & $391(21)$ & $<0.0001^{*}$ & $\begin{array}{c}2.04 \\
(1.53-2.73)\end{array}$ & $226(58)$ & $755(33)$ & $<0.0001^{*}$ & $\begin{array}{c}1.81 \\
(1.41-2.32)\end{array}$ \\
\hline $\begin{array}{l}\text { Raynaud phenomenon, } \\
\text { n }(\%)\end{array}$ & $433(94)$ & $1845(94)$ & 0.9 & NS & $329(95)$ & $1696(92)$ & 0.8 & NS & $382(99)$ & $2261(99)$ & 0.2 & NS \\
\hline Digital ulceration, n (\%) & $162(35)$ & $534(27)$ & $0.002 *$ & NS & $120(35)$ & $520(28)$ & $0.005^{*}$ & NS & $186(48)$ & $769(34)$ & $<0.0001^{*}$ & NS \\
\hline Muscle weakness, n (\%) & $189(41)$ & $505(26)$ & $<0.0001 *$ & NS & $127(37)$ & $416(22)$ & $<0.0001^{*}$ & $\begin{array}{c}1.73 \\
(1.29-2.30)\end{array}$ & $172(45)$ & $541(24)$ & $<0.0001^{*}$ & $\begin{array}{c}1.89 \\
(1.47-2.44)\end{array}$ \\
\hline Pulmonary fibrosis, n (\%) & $178(29)$ & $618(32)$ & $0.01 *$ & NS & $149(43)$ & $655(35)$ & $0.0008 *$ & NS & $178(46)$ & $829(36)$ & $0.0005^{*}$ & NS \\
\hline $\begin{array}{l}\text { Elevated systolic } \\
\text { pulmonary artery } \\
\text { pressure, n }(\%)\end{array}$ & $155(34)$ & $400(20)$ & $<0.0001^{*}$ & $\begin{array}{c}1.81 \\
(1.40-2.32)\end{array}$ & $103(30)$ & $388(21)$ & $0.0001 *$ & NS & $150(39)$ & $560(24)$ & $<0.0001^{*}$ & $\begin{array}{c}1.39 \\
(1.07-1.75)\end{array}$ \\
\hline Renal crisis, n (\%) & $21(4)$ & $54(3)$ & $0.04 *$ & NS & $15(4)$ & $31(1.5)$ & $0.02 *$ & NS & $5(6)$ & $34(1.5)$ & 0.9 & NS \\
\hline $\begin{array}{l}\text { Positive antinuclear } \\
\text { antibodies, n (\%) }\end{array}$ & $404(88)$ & $1701(87)$ & 0.8 & NS & $314(91)$ & $1704(92)$ & 0.8 & NS & $376(97)$ & $2118(93)$ & 0.2 & NS \\
\hline $\begin{array}{l}\text { Positive } \\
\text { antitopoisomerease-1 } \\
\text { antibodies, n (\%) }\end{array}$ & $202(44)$ & $636(32)$ & $<0.0001 *$ & NS & $145(42)$ & $537(29)$ & $<0.0001^{*}$ & $\begin{array}{c}1.91 \\
(1.42-2.57)\end{array}$ & $150(39)$ & $623(27)$ & $0.0003 *$ & NS \\
\hline $\begin{array}{l}\text { Positive anticentromere } \\
\text { antibodies, n (\%) }\end{array}$ & $97(21)$ & $582(30)$ & $0.0008^{*}$ & NS & $72(21)$ & $616(33)$ & $0.0003 *$ & NS & $117(30)$ & $912(40)$ & $0.0001^{*}$ & NS \\
\hline Elevated CPK, n (\%) & $69(15)$ & $218(11)$ & $0.04 *$ & NS & $34(10)$ & $128(7)$ & $0.1^{*}$ & NS & $17(4)$ & $115(5)$ & $0.1^{*}$ & NS \\
\hline $\begin{array}{l}\text { Elevation of acute- } \\
\text { phase reactants, n (\%) }\end{array}$ & $237(52)$ & $549(28)$ & $<0.001^{*}$ & $\begin{array}{c}2.29 \\
(1.81-2.90)\end{array}$ & $133(39)$ & $447(24)$ & $<0.0001^{*}$ & $\begin{array}{c}1.60 \\
(1.20-2.14)\end{array}$ & $171(44)$ & $616(27)$ & $<0.0001 *$ & $\begin{array}{c}1.49 \\
(1.16-1.91)\end{array}$ \\
\hline Proteinuria, n (\%) & $52(11)$ & $128(6)$ & $0.0004^{*}$ & NS & $24(7)$ & $99(5)$ & 0.2 & NS & $31(8)$ & $101(4)$ & $0.01 *$ & NS \\
\hline
\end{tabular}

NS: not significant; CPK: creatine phosphokinase. ${ }^{*}$ Variables included for the multivariate stepwise logistic regression analysis.

\section{DISCUSSION}

Our results highlight the striking level of articular involvement in SSc, as evaluated by systematic examination in a large cohort of patients. Overall joint involvement is more prevalent in patients with the diffuse cutaneous subtype, and is associated with more severe disease phenotype and systemic inflammation. Our data also show that synovitis, joint contracture, and tendon friction rubs are more likely to occur together.

Joint involvement has been reported to occur in a wide proportion of patients with $\mathrm{SSc}^{4}$. Many distinct abnormalities have been recognized ${ }^{4,10,22}$, but their precise prevalence in a large population of patients with SSc has not yet been accurately determined. Thus, the EUSTAR database, enabled by the major efforts of multiple medical centers, offers a unique opportunity to study these specific complications in a large population of patients with SSc. The recruitment of patients among EUSTAR centers ensured a representative population, although geographical variation of disease manifestations was recently reported ${ }^{23}$. More- over, data were standardized with the help of the MEDS and were collected in tertiary centers highly active in the field of $\mathrm{SSc}$, a situation that markedly increased their quality and accuracy.

The prevalence of clinical synovitis has not yet been determined accurately. One study systematically assessed articular involvement in 38 patients with $\mathrm{SSc}^{4}$. Tenderness, stress pain, or effusions were found in $61 \%$ of patients. Effusions, found in 10 patients (29\%), were small and predominant in the knees. The pattern was polyarticular in $61 \%$, oligoarticular in $22 \%$, and monoarticular in $17 \%$. In the EUSTAR database, synovitis was found in 1191/7286 (16\%) patients with SSc, underlining that synovial involvement may occur in SSc, although generalized arthralgia and stiffness are the more common presentations $s^{4,12,24}$. This synovial involvement has been recently reported by ultrasonography and MRI, which showed their usefulness for accurate diagnosis and characterization of synovitis of the hands and wrists of patients with SSc (Table 1) $)^{13-15}$.

The presence of synovitis may be related to an overlap Personal non-commercial use only. The Journal of Rheumatology Copyright (C) 2010. All rights reserved. 
Table 5. Disease phenotype associations in patients with SSc with or without tendon friction rubs.

\begin{tabular}{|c|c|c|c|c|c|c|c|c|c|c|c|c|}
\hline \multirow[t]{2}{*}{ Characteristic } & \multicolumn{4}{|c|}{$\begin{array}{l}\text { Whole SSc Population, } \\
\qquad \mathrm{n}=7286\end{array}$} & \multicolumn{4}{|c|}{$\begin{array}{c}\text { Patients with Diffuse Cutaneous } \\
\text { Subtype, } \mathrm{n}=2393\end{array}$} & \multicolumn{4}{|c|}{$\begin{array}{c}\text { Patients with Limited } \\
\text { Cutaneous Subtype, } n=4210\end{array}$} \\
\hline & $\begin{array}{c}\text { With } \\
\text { TFR, } \\
\mathrm{n}=802\end{array}$ & $\begin{array}{c}\text { Without } \\
\text { TFR, } \\
\mathrm{n}=6484\end{array}$ & $\mathrm{p}$ & $\begin{array}{c}\text { Stepwise } \\
\text { Regression, } \\
\text { OR } \\
(95 \% \mathrm{CI})\end{array}$ & $\begin{array}{l}\text { With } \\
\text { TFR, } \\
n=486\end{array}$ & $\begin{array}{c}\text { Without } \\
\text { TFR, } \\
\mathrm{n}=1907\end{array}$ & $\mathrm{p}$ & $\begin{array}{c}\text { Stepwise } \\
\text { Regression, } \\
\text { OR } \\
(95 \% \mathrm{CI})\end{array}$ & $\begin{array}{c}\text { With } \\
\text { TFR, } \\
\mathrm{n}=260\end{array}$ & $\begin{array}{l}\text { Without } \\
\text { TFR, } \\
\mathrm{n}=3950\end{array}$ & $\mathrm{p}$ & $\begin{array}{c}\text { Stepwise } \\
\text { Regression } \\
\text { OR } \\
(95 \% \mathrm{CI})\end{array}$ \\
\hline Age, mean $\pm \mathrm{SD}, \mathrm{yrs}$ & $56 \pm 15$ & $58 \pm 18$ & $0.001^{*}$ & NS & $51 \pm 13$ & $55 \pm 15$ & $0.002 *$ & $\begin{array}{c}0.69 \\
(0.48-0.85)\end{array}$ & $59 \pm 15$ & $60 \pm 19$ & 0.3 & NS \\
\hline $\begin{array}{l}\text { Disease duration, } \\
\text { mean } \pm \mathrm{SD} \text {, yrs }\end{array}$ & $9 \pm 10$ & $10 \pm 11$ & 0.53 & NS & $4 \pm 8$ & $9 \pm 10$ & $0.0001^{*}$ & $\begin{array}{c}0.56 \\
(0.32-0.83)\end{array}$ & $10 \pm 11$ & $10 \pm 12$ & 0.9 & NS \\
\hline Synovitis, n (\%) & $291(36)$ & 900 (14) & $<0.0001^{*}$ & $\begin{array}{c}2.31 \\
(1.91-2.79)\end{array}$ & $173(36)$ & $311(16)$ & $<0.0001 *$ & $\begin{array}{c}2.20 \\
(1.69-2.87)\end{array}$ & $93(36)$ & $477(12)$ & $<0.0001^{*}$ & $\begin{array}{c}2.90 \\
(2.18-3.96)\end{array}$ \\
\hline Joint contracture, $\mathrm{n}(\%)$ & $528(66)$ & $1736(27)$ & $<0.0001 *$ & $\begin{array}{c}3.04 \\
(2.54-3.65)\end{array}$ & $365(75)$ & $802(42)$ & $<0.0001 *$ & $\begin{array}{c}3.15 \\
(2.44-4.06)\end{array}$ & $139(53)$ & $836(21)$ & $<0.0001^{*}$ & $\begin{array}{c}2.66 \\
(2.01-3.54)\end{array}$ \\
\hline Muscle weakness, n (\%) & $367(46)$ & $1583(24)$ & $<0.0001 *$ & $\begin{array}{c}1.42 \\
(1.18-1.70)\end{array}$ & $245(50)$ & $625(33)$ & $<0.0001 *$ & $\begin{array}{c}1.32 \\
(1.03-2.14)\end{array}$ & $103(40)$ & $801(20)$ & $<0.0001^{*}$ & $\begin{array}{c}1.84 \\
(1.38-2.46)\end{array}$ \\
\hline Pulmonary fibrosis, n (\%) & $399(50)$ & $2208(34)$ & $<0.0001^{*}$ & $\begin{array}{c}1.22 \\
(1.02-1.46)\end{array}$ & $278(57)$ & $939(49)$ & $0.003^{*}$ & NS & $106(41)$ & $1110(28)$ & $<0.0001^{*}$ & $\begin{array}{c}1.49 \\
(1.12-1.98)\end{array}$ \\
\hline Elevated sPAP, n (\%) & $272(34)$ & $1484(23)$ & $<0.0001^{*}$ & NS & $175(36)$ & $463(24)$ & $<0.0001^{*}$ & NS & $78(30)$ & $874(22)$ & $0.02 *$ & NS \\
\hline Renal crisis, n (\%) & $36(4)$ & $124(2)$ & $<0.0001 *$ & NS & $27(6)$ & $74(4)$ & $0.1 *$ & NS & $7(3)$ & $42(1)$ & $0.03 *$ & NS \\
\hline Positive ANA, n (\%) & $740(92)$ & $5877(91)$ & 0.78 & NS & $444(91)$ & $1727(91)$ & 0.4 & NS & $234(90)$ & $3629(92)$ & 0.6 & NS \\
\hline $\begin{array}{l}\text { Positive } \\
\text { antitopoisomerease-1 } \\
\text { antibodies, n (\%) }\end{array}$ & $382(48)$ & $1911(29)$ & $<0.0001 *$ & NS & $291(60)$ & $1026(54)$ & $0.001^{*}$ & NS & $95(37)$ & $763(19)$ & $0.0001^{*}$ & $\begin{array}{c}1.32 \\
(1.15-1.72)\end{array}$ \\
\hline $\begin{array}{l}\text { Positive anticentromere } \\
\text { antibodies, } \mathrm{n}(\%)\end{array}$ & $130(16)$ & $2266(35)$ & $<0.0001^{*}$ & NS & $21(4)$ & $119(6)$ & 0.2 & NS & $101(39)$ & $1950(49)$ & $0.001 *$ & NS \\
\hline
\end{tabular}

TFR: tendon friction rubs; NS: not significant; CPK: creatine phosphokinase; ANA: antinuclear antibodies; SSc: systemic sclerosis; sPAP: systolic pulmonary artery pressure.

* Variables included for the multivariate stepwise logistic regression analysis.

with rheumatoid arthritis (RA) or, more probably, to the existence of primary erosive arthropathy specific to $\mathrm{SSc}^{9,25-27}$. Recent data indicate that the overlap of SSc and RA is unusual. The prevalence of SSc-RA overlap is $1 \%$ to $5 \%$ and its incidence is $5 \%{ }^{17,28-30}$. Moreover, 1 study from our group showed in a population of 120 patients with SSc a point prevalence of radiographic erosive arthritis of $18 \%$, while only 2 patients with SSc $(2 \%)$ with radiographic arthritis fulfilled the American College of Rheumatology criteria for classic $\mathrm{RA}^{9}$. These data are supported by the low frequency of antibodies against cyclic citrullinated peptide in SSc (prevalence 1.5\% to 8\%), which have the highest specificity for the diagnosis of $\mathrm{RA}^{31-33}$.

Patients with synovitis and early disease were more likely to experience diffuse cutaneous thickening. This observation raises the possibility of using synovitis in patients with early SSc to identify those with potential risk of developing the diffuse cutaneous subset, which has a more fulminant course. We also found that the likelihoods of severe vascular and muscular involvement were higher in patients with synovitis, regardless of their cutaneous subset or their disease duration. Thus, synovitis could be a risk factor of bad prognosis in SSc, and we suggest that all patients be screened for synovitis immediately after the diagnosis of SSc. The validation of synovitis as a predictive factor of the diffuse cutaneous subset and bad prognosis is now under investigation in the prospective followup of patients with SSc included in the EUSTAR database.

We found that elevation of acute-phase reactants, reflecting systemic inflammation, was strongly associated with synovitis. This suggests that joint involvement has a close relationship with systemic inflammation in $\mathrm{SSc}^{9}$. This is supported by the existence of inflammatory cell infiltration, associated with focal microvascular obliteration and fibrin deposition, on synovial biopsies performed on patients with $\mathrm{SSc}^{34}$. Further studies are warranted to determine whether 
Table 6. Disease characteristics associated with tendon friction rubs, according to disease duration.

\begin{tabular}{|c|c|c|c|c|c|c|c|c|c|c|c|c|}
\hline \multirow[t]{2}{*}{ Characteristic } & \multicolumn{4}{|c|}{$\begin{array}{l}\text { Patients with Disease Duration, } \\
\quad<5 \text { Years, } \mathrm{n}=2421\end{array}$} & \multicolumn{4}{|c|}{$\begin{array}{l}\text { Patients with Disease Duration } \\
\text { Between } 5 \text { and } 10 \text { Years, } \mathrm{n}=2196\end{array}$} & \multicolumn{4}{|c|}{$\begin{array}{l}\text { Patients with Disease Duration } \\
\qquad>10 \text { Years, } \mathrm{n}=2669\end{array}$} \\
\hline & $\begin{array}{c}\text { With } \\
\text { TFR, } \\
\mathrm{n}=322\end{array}$ & $\begin{array}{c}\text { Without } \\
\text { TFR, } \\
\mathrm{n}=2099\end{array}$ & $\mathrm{p}$ & $\begin{array}{c}\text { Stepwise } \\
\text { Regression, } \\
\text { OR } \\
(95 \% \mathrm{CI})\end{array}$ & $\begin{array}{c}\text { With } \\
\text { TFR, } \\
\mathrm{n}=213\end{array}$ & $\begin{array}{c}\text { Without } \\
\text { TFR, } \\
\mathrm{n}=1983\end{array}$ & $\mathrm{p}$ & $\begin{array}{c}\text { Stepwise } \\
\text { Regression, } \\
\text { OR } \\
(95 \% \mathrm{CI})\end{array}$ & $\begin{array}{c}\text { With } \\
\text { TFR, } \\
\mathrm{n}=267\end{array}$ & $\begin{array}{c}\text { Without } \\
\text { TFR, } \\
\mathrm{n}=2402\end{array}$ & $\mathrm{p}$ & $\begin{array}{c}\text { Stepwise } \\
\text { Regression } \\
\text { OR } \\
(95 \% \mathrm{CI})\end{array}$ \\
\hline Age, mean $\pm \mathrm{SD}$, yrs & $51 \pm 10$ & $56 \pm 11$ & $0.001 *$ & $\begin{array}{c}0.71 \\
(0.48-0.92)\end{array}$ & $55 \pm 13$ & $57 \pm 10$ & $0.1^{*}$ & NS & $58 \pm 12$ & $61 \pm 14$ & $0.009 *$ & NS \\
\hline $\begin{array}{l}\text { Diffuse cutaneous, } \\
\text { subtype, } \mathrm{n}(\%)\end{array}$ & $230(71)$ & $787(37)$ & $<0.0001 *$ & $\begin{array}{c}2.58 \\
(1.87-3.53)\end{array}$ & $126(59)$ & $509(26)$ & $<0.0001^{*}$ & $\begin{array}{c}1.54 \\
(1.09-2.18)\end{array}$ & $130(49)$ & $611(25)$ & $<0.0001^{*}$ & $\begin{array}{c}2.09 \\
(1.52-2.89)\end{array}$ \\
\hline Synovitis, n (\%) & $121(38)$ & $339(16)$ & $<0.0001^{*}$ & $\begin{array}{c}2.80 \\
(2.03-3.81)\end{array}$ & $66(31)$ & $279(14)$ & $<0.0001^{*}$ & $\begin{array}{c}2.54 \\
(1.97-3.67)\end{array}$ & $104(39)$ & $282(12)$ & $<0.0001 *$ & $\begin{array}{c}2.85 \\
(2.04-3.97)\end{array}$ \\
\hline Joint contracture, $\mathrm{n}(\%)$ & $196(61)$ & $549(26)$ & $<0.0001^{*}$ & $\begin{array}{c}2.41 \\
(1.78-3.23)\end{array}$ & $146(69)$ & $392(20)$ & $<0.0001^{*}$ & $\begin{array}{c}4.55 \\
(3.20-6.47)\end{array}$ & $186(70)$ & $795(33)$ & $<0.0001^{*}$ & $\begin{array}{c}3.05 \\
(2.18-4.27)\end{array}$ \\
\hline Muscle weakness, n (\%) & $150(47)$ & $544(26)$ & $<0.0001^{*}$ & $\begin{array}{c}1.37 \\
(1.02-1.86)\end{array}$ & $95(45)$ & $448(23)$ & $<0.0001^{*}$ & $\begin{array}{c}1.61 \\
(1.14-2.27)\end{array}$ & $122(46)$ & $591(25)$ & $<0.0001^{*}$ & $\begin{array}{c}1.54 \\
(1.12-2.13)\end{array}$ \\
\hline Pulmonary fibrosis, n (\%) & $149(46)$ & $647(31)$ & $<0.0001^{*}$ & NS & $120(56)$ & $684(34)$ & $<0.0001^{*}$ & $\begin{array}{c}1.55 \\
(1.11-2.18)\end{array}$ & $130(49)$ & $877(37)$ & $<0.0001^{*}$ & NS \\
\hline Elevated sPAP, n (\%) & $102(32)$ & $453(22)$ & $0.0006^{*}$ & NS & $66(31)$ & $425(21)$ & $0.005^{*}$ & $\mathrm{NS}$ & $104(39)$ & $606(25)$ & $<0.0001 *$ & $\begin{array}{c}1.60 \\
(1.16-2.21)\end{array}$ \\
\hline Renal crisis, n (\%) & $17(5)$ & $58(3)$ & $0.02 *$ & NS & $9(4)$ & $37(2)$ & $0.03 *$ & NS & $10(4)$ & $29(1)$ & $0.02 *$ & NS \\
\hline Positive ANA, n (\%) & $296(92)$ & $1809(86)$ & 0.7 & NS & $192(90)$ & $1826(92)$ & 0.8 & NS & $252(94)$ & $2242(93)$ & 0.8 & NS \\
\hline $\begin{array}{l}\text { Positive sc170 } \\
\text { antibodies, } \mathrm{n}(\%)\end{array}$ & $164(51)$ & $674(32)$ & $<0.0001^{*}$ & NS & $103(48)$ & $579(29)$ & $<0.0001 *$ & NS & $115(43)$ & $658(27)$ & $<0.0001^{*}$ & NS \\
\hline Positive ACA, n (\%) & $39(12)$ & $640(30)$ & $<0.0001^{*}$ & NS & $32(15)$ & $656(33)$ & $<0.0001^{*}$ & NS & $59(22)$ & $970(40)$ & $<0.0001^{*}$ & NS \\
\hline Elevated CPK, n (\%) & $72(22)$ & $215(10)$ & $<0.0001 *$ & NS & $26(12)$ & $136(7)$ & $0.007 *$ & NS & $17(6)$ & $115(5)$ & $0.09^{*}$ & NS \\
\hline
\end{tabular}

TFR: tendon friction rubs; NS: not significant; CPK: creatine phosphokinase; ANA: antinuclear antibodies; ACA: anticentromere antibodies; sPAP: systolic pulmonary artery pressure. * Variables included for the multivariate stepwise logistic regression analysis.

joint involvement is the main contributor to systemic inflammation in SSc (as in RA).

Tendon friction rubs are also common during the disease course, leading to a coarse and palpable crepitus, which is very specific to SSc. These rubs were found in $11 \%$ of this large cohort and were more prevalent in patients with the diffuse cutaneous subset and early disease. However, their prevalence was lower than previously reported in a large American study of 1305 patients with SSc. In that study, rubs were found in $28 \%(368 / 1305)$ of patients ${ }^{35}$. This different point prevalence could be partly explained by the higher proportion of patients with the diffuse cutaneous subset in the American study (49\% vs 33\% in our study).

As expected, we found that friction rubs were associated with the diffuse cutaneous subtype in all disease stages, but with higher OR in the subset of patients with early disease. This observation underlines the strength of association between rubs and diffuse cutaneous SSc in patients with early disease, as observed in the American study ${ }^{35}$.
In patients with the diffuse cutaneous subset and with early disease, the presence of tendon friction rubs was associated with signs of severe vascular, muscular, and renal involvement, as reported ${ }^{10,15,24}$. This is also supported by a study that assessed the clinical and prognostic significance of palpable tendon friction rubs in patients with SSc. The authors showed strong correlations between the presence of tendon friction rubs and typical features of diffuse cutaneous SSc: more severe skin thickening, more frequent heart and kidney involvement, and decreased survival ${ }^{35}$.

One original point raised by our results is the identification of patients with rubs and limited cutaneous subtype or with rubs and late disease as new groups at risk of severe disease. It is noteworthy that patients with friction rubs and limited cutaneous disease had a higher frequency of antitopoisomerase I antibodies. This observation suggests that these patients may represent a subset of individuals with "subclinical" or "aborted" diffuse SSc. Altogether, our data suggest that searching for friction rub should be a routine 
Table 7. Disease phenotype associations in patients with SSc with or without joint contracture (JC).

\begin{tabular}{|c|c|c|c|c|c|c|c|c|c|c|c|c|}
\hline \multirow[t]{2}{*}{ Characteristic } & \multicolumn{4}{|c|}{$\begin{array}{l}\text { Whole SSc Population, } \\
\qquad \mathrm{n}=7286\end{array}$} & \multicolumn{4}{|c|}{$\begin{array}{l}\text { Patients with Diffuse Cutaneous } \\
\text { Subtype, } \mathrm{n}=2393\end{array}$} & \multicolumn{4}{|c|}{$\begin{array}{c}\text { Patients with Limited } \\
\text { Cutaneous Subtype, } n=4210\end{array}$} \\
\hline & $\begin{array}{c}\text { With } \\
\text { JC, } \\
\mathrm{n}=2264\end{array}$ & $\begin{array}{c}\text { Without } \\
\text { JC, } \\
\mathrm{n}=5022\end{array}$ & $\mathrm{p}$ & $\begin{array}{c}\text { Stepwise } \\
\text { Regression, } \\
\text { OR } \\
(95 \% \mathrm{CI})\end{array}$ & $\begin{array}{c}\text { With } \\
\text { JC, } \\
\mathrm{n}=1167\end{array}$ & $\begin{array}{c}\text { Without } \\
\text { JC, } \\
\mathrm{n}=1226\end{array}$ & $\mathrm{p}$ & $\begin{array}{c}\text { Stepwise } \\
\text { Regression, } \\
\text { OR } \\
(95 \% \mathrm{CI})\end{array}$ & $\begin{array}{c}\text { With } \\
\text { JC, } \\
\mathrm{n}=975\end{array}$ & $\begin{array}{l}\text { Without } \\
\text { JC, } \\
\mathrm{n}=3235\end{array}$ & $\mathrm{p}$ & $\begin{array}{c}\text { Stepwise } \\
\text { Regression } \\
\text { OR } \\
(95 \% \mathrm{CI})\end{array}$ \\
\hline Age, mean $\pm \mathrm{SD}$, yrs & $57 \pm 16$ & $58 \pm 17$ & $0.01 *$ & NS & $53 \pm 14$ & $56 \pm 15$ & $0.04 *$ & NS & $58 \pm 15$ & $62 \pm 16$ & $0.001 *$ & NS \\
\hline Friction rub, n (\%) & $528(23)$ & $274(5)$ & $<0.0001 *$ & $\begin{array}{c}2.89 \\
(2.39-3.50)\end{array}$ & $365(31)$ & $121(10)$ & $<0.0001 *$ & $\begin{array}{c}3.16 \\
(2.42-4.12)\end{array}$ & $133(14)$ & $134(4)$ & $<0.0001^{*}$ & $\begin{array}{c}* 2.67 \\
(1.98-3.61)\end{array}$ \\
\hline Synovitis, n (\%) & $587(26)$ & $604(12)$ & $<0.0001 *$ & $\begin{array}{c}1.75 \\
(1.49-2.05)\end{array}$ & $302(26)$ & $182(15)$ & $<0.0001 *$ & $\begin{array}{c}1.53 \\
(1.18-1.98)\end{array}$ & $233(24)$ & $337(10)$ & $<0.0001^{*}$ & $* \begin{array}{c}2.01 \\
(1.68-2.59)\end{array}$ \\
\hline $\begin{array}{l}\text { Raynaud phenomenon, } \\
\mathrm{n}(\%)\end{array}$ & $2172(96)$ & $4774(95)$ & 0.2 & NS & $1118(96)$ & $1170(95)$ & 0.9 & NS & $933(96)$ & $3106(96)$ & $0.1^{*}$ & NS \\
\hline $\begin{array}{l}\text { Pulmonary fibrosis, } \\
\mathrm{n}(\%)\end{array}$ & $1050(46)$ & $1557(31)$ & $<0.0001^{*}$ & $\begin{array}{c}1.23 \\
(1.08-1.39)\end{array}$ & $623(53)$ & $594(48)$ & $0.02 *$ & NS & $371(38)$ & $845(26)$ & $<0.0001^{*}$ & NS \\
\hline Elevated sPAP, n (\%) & $715(32)$ & $1041(21)$ & $<0.001^{*}$ & $\begin{array}{c}1.38 \\
(1.19-1.58)\end{array}$ & $373(32)$ & $259(21)$ & $<0.0001 *$ & $\begin{array}{c}1.47 \\
(1.17-1.84)\end{array}$ & $287(29)$ & $665(21)$ & $<0.0001^{*}$ & $* \begin{array}{c}* 1.30 \\
(1.07-1.57)\end{array}$ \\
\hline Renal crisis, n (\%) & $72(3)$ & $88(2)$ & $0.0001 *$ & NS & $53(5)$ & $48(4)$ & 0.5 & NS & $16(2)$ & $33(1)$ & $0.1^{*}$ & NS \\
\hline $\begin{array}{l}\text { Positive antinuclear } \\
\text { antibodies, } \mathrm{n}(\%)\end{array}$ & $2103(93)$ & $4514(90)$ & $0.06^{*}$ & NS & $1080(93)$ & $1091(89)$ & $0.03^{*}$ & NS & $878(90)$ & $2985(92)$ & 0.9 & NS \\
\hline $\begin{array}{l}\text { Positive } \\
\text { antitopoisomerease-1 } \\
\text { antibodies, n (\%) }\end{array}$ & $975(43)$ & $1318(26)$ & $<0.0001 *$ & NS & $688(59)$ & $629(51)$ & $0.0004 *$ & NS & $261(27)$ & $597(18)$ & $<0.0001^{*}$ & NS \\
\hline $\begin{array}{l}\text { Positive anticentromere } \\
\text { antibodies, } \mathrm{n}(\%)\end{array}$ & 406 (18) & $1990(40)$ & $<0.0001 *$ & $\begin{array}{c}0.56 \\
(0.47-0.66)\end{array}$ & $48(4)$ & $92(8)$ & $0.0009^{*}$ & $\begin{array}{c}0.59 \\
(0.39-0.90)\end{array}$ & $340(35)$ & $1711(53)$ & $<0.0001^{*}$ & $\begin{array}{c}* \quad 0.50 \\
(0.42-0.59)\end{array}$ \\
\hline
\end{tabular}

NS: not significant; CPK: creatine phosphokinase; sPAP: systolic pulmonary artery pressure. * Variables included for the multivariate stepwise logistic regression analysis.

part of the physical examination also in patients with the limited cutaneous subset or in a late disease stage. The predictive value of tendon friction rubs in these new groups at risk will be further investigated with the ongoing prospective followup of patients entered in the EUSTAR database.

Joint contracture results from joint destruction, turning into ankylosis, and fibrotic changes in the skin, the hallmark of SSc and a source of functional disability ${ }^{6,7}$. We confirm that joint contracture is associated with the diffuse cutaneous subset in all disease stages, known to have the higher fibrotic propensity, and with pulmonary fibrosis. This association, taken with the association we reported between flexion contracture and Health Assessment Questionnaire findings ${ }^{9}$, emphasizes the greater impairment of hand function in the diffuse subgroup, as reported by Brower and Poole ${ }^{6}$. We did not find any association in multivariate analysis between the serum levels of antitopoisomerase I antibodies and joint flexion contracture, in contrast with recently published data that have suggested a high correlation ${ }^{36}$.
Our data show that synovitis, joint contracture, and tendon friction rubs are strongly associated in the multivariate analysis. This suggests an interdependence of these symptoms in the development of articular involvement and further disability. This may also indicate a shared mechanism in their development.

The frequency of any articular symptoms was $28 \%$ (2025/7286) in this large cohort, which is consistent with other findings $\mathrm{s}^{4,10,12}$ and highlights the frequency and the burden of articular involvement in SSc. Indeed, after lifethreatening complications, important articular involvement may weigh on the quality of life of patients with SSc, as has been demonstrated ${ }^{5,9}$. As for synovitis, tendon friction rubs, or joint contracture, the occurrence of any joint symptoms was associated with a severe disease phenotype, suggesting that joint involvement could be a potential marker of disease severity.

Our study is limited by its observational design, and any pathogenic link emerging from this type of study should be 
Table 8. Disease characteristics associated with joint contracture (JC), according to disease duration.

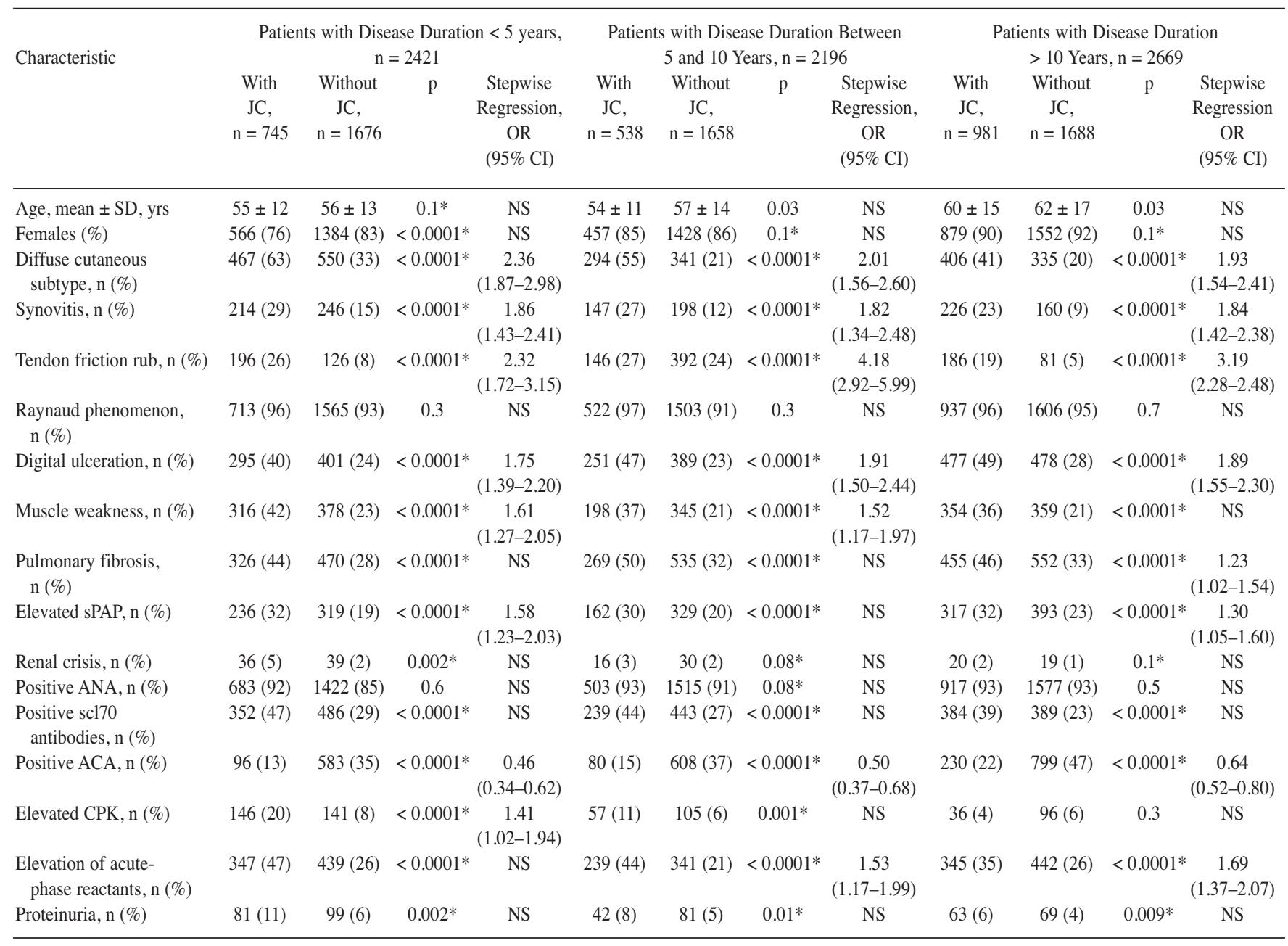

NS: not significant; CPK: creatine phosphokinase; sPAP: systolic pulmonary artery pressure; ANA: antinuclear antibodies; ACA: anticentromere antibodies. * Variables included for the multivariate stepwise logistic regression analysis.

regarded cautiously. We expect the prospective followup of this cohort to strengthen the associations we found and to assess articular involvement as a marker and a predictor of disease severity. The association between joint involvement and elevated SPAP should be regarded cautiously, as there was no definite pulmonary arterial hypertension confirmation with right-heart catheterization. The difficulty of detecting synovitis because of overlying cutaneous thickening and the difficulty of distinguishing synovitis from joint pain with swollen fingers may have biased the prevalence of synovitis. Moreover, most of our patients were postmenopausal women and were therefore prone to develop osteoarthritis, which could be characterized by tender and swollen joints. We could not rule out the possibility of such an arthropathy, unrelated to SSc, occurring in our patients.

The brevity and shallowness of the data collected in the MEDS form did not allow us to assess the precise distribution of joint involvement [number and localization of tender/swollen joints and friction rubs, joint(s) involved in joint contracture]. This contrasts with studies performed on a limited number of patients, but with a more in-depth view of the extent, frequency, and degree of synovitis, friction rubs, and contracture coupled with radiographs, ultrasound, and MRI results.

We also could not assess the contribution of articular manifestations to disability, or the influence of other measurements on joint involvement, such as therapies (corticosteroids, disease-modifying drugs), specific antibodies (rheumatoid factors, anti-citrullinated peptide antibodies/ second generation), or potential overlap with other diseases, such as RA. The results of radiographs were not collected in the database, which prevented us from correlating clinical and radiological joint involvement.

We did not perform any analysis between joint involvement and disease activity. The European disease activity index, proposed by the European Scleroderma Study Group, was collected in the MEDS and recorded as present if it displayed a score $\geq 3^{37}$. However, the calculation of the final 
score included synovitis and increased ESR. As it was not possible to analyze this index independently from these 2 items, we did not perform any analysis between joint involvement and disease activity. The current development of the MEDS Online database, with a huge collection of data and a prospective followup, will allow us to complete this first evaluation.

Our results highlight the striking level of articular involvement in $\mathrm{SSc}$, as evaluated by systematic examination in a large cohort of patients with SSc. Our study also shows that articular involvement is associated with more severe disease and with systemic inflammation. This observation suggests that the subset of patients with SSc who have articular symptoms may be regarded as a severe disease subgroup, and the predictive values of articular involvement on outcomes remain be determined.

\section{ACKNOWLEDGMENT}

EUSTAR Coauthors: I. Miniati, Department of BioMedicine, Division of Rheumatology AOUC, University of Florence, Italy; A. Müller, Department of Internal Medicine-I, University of Regensburg, Regensburg, Germany; F. Iannone, U.O. Reumatologia Università degli studi di Bari, Az. Ospedaliera Policlinico Padiglione V. Chini, di Bari, Bari, Italy; R. Giacomelli, Dipartimento di Medicina Interna e Sanità Pubblica, Insegnamento di Reumatologia, Servizio per lo Studio, diagnosi e cura delle malattie immunologiche, University di L'Aquila, L'Aquila, Italy; O. Distler, Department of Rheumatology, University Hospital Zurich, Zurich, Switzerland; R. Becvar, Institute of Rheumatology, 1st Medical School, Charles University, Prague, Czech Republic; S. Sierakowsky; O. KowalBielecka, Department of Rheumatology and Internal Diseases, Medical University of Bialystok, Bialystok, Poland; P. Coelho, Instituto portugues de Reumatologia, Lisboa, Portugal; J. Cabane, Service de Médecine Interne, Hopital Saint Antoine, Paris, France; M. Cutolo, Research Laboratory and Division of Rheumatology, Department of Internal Medicine, University of Genova, Genova, Italy; Y. Shoenfeld, Center for Autoimmune Diseases, Department of Medicine B, Sackler Faculty of Medicine, Tel-Aviv University, Tel-Aviv, Israel; G. Valentini, Dipartimento Medicina Clinica e Sperimentale "F. Magrassi," II Policlinico U.O. Reumatologia, Napoli, Italy; J. Rovensky, Institute of Rheumatic Diseases, Piestany Národny ústav Reumatickych Chorôb, Piestany, Slovak Republic; G. Riemekasten, Department of Rheumatology - Charité University Hospital, Berlin, Germany; I. Nicoara, Clinica Reumatologie - University of Medicine and Pharmacy "iuliu Hatieganu" Cluj, Cluj-Napoca, România; P. Vlachoyiannopoulos, Department of Pathopysiology Medical School, National University of Athens, Athens, Greece; R. Caporali, Unità Operativa e Cattedra di Reumatologia, IRCCS Policlinico S, Matteo, Pavia, Italy; S. Jiri, Department of Dermatology, 1st Faculty of Medicine, Charles University, Prague, Czech Republic; M. Inanc, Department of Internal Medicine, Division of Rheumatology, Istanbul Medical Faculty, Istanbul, Turkey; I. Zimmermann Gorska, Department of Rheumatology and Rehabilitation, Karol Marcinkowski University of Medical Sciences, Poznan, Poland; P. Carreira, Servicio de Reumatología, Hospital Universitario 12 de Octubre, Madrid, Spain; S. Novak, Department of Rheumatology and Clinical Immunology, Internal Medicine, KBC Rijeka, Croatia; L. Czirjak, Department of Immunology and Rheumatology, Faculty of Medicine, University of Pecs, Pecs, Hungary; F. Oliveira Ramos, Department of Rheumatology, Hospital Santa Maria, Lisbon, Portugal; M. Jendro, Rheumatologische Ambulanz, Medizinische Klinik I, Universitätskliniken des Saarlandes, Homburg, Germany; C. Chizzolini, Immunology and Allergy, University Hospital, Geneva, Switzerland; E.J. Kucharz, Department of Internal Medicine and Rheumatology, Medical
University of Silesia, Katowice, Poland; J. Richter, Rheumatology Department, Heinrich-Heine University, Düsseldorf, Germany; F. Cozzi, Division of Rheumatology, Department of Medical and Surgical Sciences, University of Padova, Padova, Italy; B. Rozman, University Medical Center Ljublijana, Division of Internal Medicine, Department of Rheumatology, Ljubljana, Slovenia; C.M. Mallia, "Stella Maris,” Balzan, Malta; A. Gabrielli, Istituto di Clinica Medica Generale, Ematologia ed Immunologia, Clinica Polo Didattico, Università Politecnica delle Marche, Ancona, Italy; D. Farge, Department of Internal Medicine, Hopital Saint Louis, Paris, France; H.P. Kiener, Department of Rheumatology, Internal Medicin III, University of Vienna, Vienna, Austria; D. Schöffel, Westpfalz-Klinikum, Department of Rheumatology, Kusel, Germany; M. Sticherling, Klinik für Dermatologie, Venerologie und Allergologie, Universitätsklinikum Leipzig, Leipzig, Germany; P. Airo, Servizio di Reumatologia e Immunologia Clinica, Spedali Civili, Brescia, Itali; F. Wollheim, Department of Rheumatology, Lund University Hospital, Lund, Sweden; D. Martinovic, Rheumatology Department of Internal Clinic, Clinical Hospital of Split, Split, Croatia; F. Trotta, Department Of Clinical and Experimental Medicine, Section of Rheumatology, University of Ferrara, Ferrara, Italy; N. Hunzelmann Universitätshautklinik Köln, Köln, Germany; S. Jablonska, Department of Dermatology, Warsaw, Poland; K. Reich, Department of Dermatology, Georg-August-University Göttingen, Göttingen, Germany; S. Bombardieri, Department of Internal Medicine, Rheumatology Unit, University of Pisa-Santa Chiara, Pisa, Italy; P. Siakka, Department of Rheumatology, General Hospital "Ag. Pavlos," Thessaloniki, Greece; R. Pellerito, Ospedale Mauriziano, Centro di Reumatologia, Torino, Italy; L.M. Bambara, Dipartimento di Medicina Clinica e Sperimentale, Reumatologia-Medicina Interna B, Università degli Studi di Verona, Verona, Italy; J. Morovic-Vergles, Division of Clinical Immunology and Rheumatology, Department of Internal Medicine, Dubrava University Hospital, Zagreb, Croatia; C. Denton, Centre for Rheumatology, Royal Free and University College London Medical School, London, United Kingdom; R. Hinrichs, Klinik für Dermatologie und Allergologie, Universität Ulm, Ulm, Germany; F. Van den Hoogen, Universitair Medisch Centrum St. Radboud, Nijmegen, The Netherlands; N. Damjanov, Institute of Rheumatology, Belgrade, Serbia and Montenegro; I. Kötter, Universitätsklinikum Tübingen, Medizinische Klinik und Poliklinik, Tübingen, Germany; V. Ortiz, Rheumatology Granollers General Hospital, Barcelona, Spain; S. Heitmann, Department of Rheumatology, Marienhospital Stuttgart, Stuttgart, Germany; D. Krasowska, Department of Dermatology, Medical University of Lublin, Lublin, Poland; M. Seidel, Department of Rheumatology, Medizinische Universitäts-Poliklinik, Bonn, Germany; P. Hasler, Kantonsspital Aarau, Rheumaklinik und Institut für Physikalische Medizin und Rehabilitation, Aarau, Switzerland; J.M. Van Laar, Department of Rheumatology, Leiden University Medical Center, Leiden, The Netherlands; J.P. Kaltwasser, Klinikum der Johan Wolfgang Goethe, Universität, Medizinische Klinik III, Rheumatologische Ambulanz, Frankfurt, Germany; I. Foeldvari, Kinder- und Jugendrheumatologie, Hamburger Zentrum für Kinder- und Jugendrheumatologie, Am Klinikum Eilbek, Hamburg, Germany; A. Juan Mas, Hospital son llàtzer, Palma de Mallorca, Spain; G. Bajocchi, Arcispedale Santa Maria Nuova, Dipartimento Area Medica 1, UO di Reumatologia, Reggio Emilia, Italy; M. Wislowska, Department of Rheumatology, Central Clinical Hospital MSW I A, Warsaw, Poland; J.A. Pereira Da Silva, Reumatologia, Hospitais da Universidade, Coimbra, Portugal; S. Jacobsen, Department of Rheumatology, Rigshospitalet, University of Copenhagen, Copenhagen, Denmark; M. Worm, Department of Dermatology and Allergy, Charité - Universitätsmedizin Berlin, Berlin, Germany; W. Graniger, Medizinische Universität Graz, Medizinische Universitätsklinik - Abteilung für Rheumatologie, Graz, Austria; A. Kuhn, Department of Dermatology, University of Düsseldorf, Düsseldorf, Germany; A. Stankovic, Institute for Prevention, Treatment and Rehabilitation, Rheumatic and Cardiovascular Disease, Niska Banja, Serbia and Montenegro; R. Cossutta, Rheumatology Unit - Humanitas Clinical Institute, Rozzano (Milan), Italy; M. Majdan, Department of 
Reumatology and Connective Tissue Diseases, Medical University of Lublin, Lublin, Poland; L. Damjanovska Rajcevska, Clinical Center Skopje, Rheumatology Clinic "Dr Dimitar Arsov," University St. Cyril and Methodius, Skopje, FYR Macedonia; M. Tikly, Rheumatology Unit, Department of Medicine, Chris Hani Baragwanath Hospital and University of the Witwatersrand, Johannesburg, South Africa; E.L. Nasonov, Institute of Rheumatology, Russian Academy of Medical Science, Moscow, Russia; K. Steinbrink, Department of Dermatology, University of Mainz, Mainz, Germany; A. Herrick, Hope Hospital/University of Manchester, Rheumatic Diseases Centre, Salford, United Kingdom; U. Müller-Ladner, Kerckhoff-Klinik Bad Nauheim, Bad Nauheim, Germany; A. Dinc, Gulhane Military Medical Academy Division of Rheumatology, Etlik-Ankara, Turkey; R. Scorza, UO Immunologia Clinica, Centro di Riferimento per le Malattie Autoimmuni Sistemiche, Milano, Italy; K. Sondergaard, Department of Rheumatology, Aarhus University Hospital, Aarhus, Denmark; F. Indiveri, Clinica di Medicina Interna Ad Orientamento Immunologico, Università di Genova, Genova, Italy; H. Nielsen, Department of Rheumatology and Endocrinology, Herlev, Denmark; Z. Szekanecz, Third Department of Medicine, Rheumatology Division, University of Debrecen, Medical Center, Hungary; R.M. Silver, Division of Rheumatology and Immunology, Charleston, South Carolina, USA; M. Antivalle, Ospedale L, Sacco - Azienda Ospedaliera-Polo Universitario, Unità Operativa di Reumatologia, Milano, Italy; I.B. Espinosa, Consulta Reumatologia Hospital deMendaro, Mendaro Guipuzcoa, Spain; P. García de la Pena Lefebvre, Servicio de Reumatología, Hospital Ramon Y Cajal, Madrid, Spain; O. Midtvedt, Department of Rheumatology, Rikshospitalet University Hospital, Oslo, Norway; D. Launay, Department of Internal Medicine, Hôpital Claude Huriez, Lille, France; F. Valesini, Divisione Di Reumatologia - Universita' Di Roma La Sapienza, Roma, Italy; P. Tuvik, North-Estonian Regional Hospital, Tallinn, Estonia; R.M. Ionescu, Department of Internal Medicine and Rheumatology, "Carol Davila" University of Medicine and Pharmacy, Bucharest, Romania; N. Del Papa, Rheumatology, Gaetano Pini, Milano, Italy; S. Pinto, Serviço de Reumatologia, Hospital São João, Porto, Portugal; F. Wigley, Division of Rheumatology, Johns Hopkins University, Johns Hopkins School of Medicine, Baltimore, MD, USA; C. Mihai, Clinic of Internal Medicine and Rheumatology, Dr I Cantacuzino Hospital and Early Arthritis Center, Bucharest, Romania; M. Sinziana Capranu, Department of Rheumatology, Dr. D. Gerota Emergency Hospital, Bucharest, Romania; C. Sunderkötter, Department of Dermatology, University of Münster, Münster, Germany; J.B. Jun, Hanyang University, Hospital for Rheumatic Diseases, Seoul, Korea; C. Derk, Thomas Jefferson University, Philadelphia, PA, USA; S. Alhasani, Rheumatology and Rehabilitation Department, Ibnsina Teaching Hospital, Mosul, Iraq; J.H. Distler, Department of Internal Medicine 3, Erlangen, Germany; E. Ton, University Medical Centre Utrecht, Utrecht, The Netherlands; T. Soukup, 2nd Department of Internal Medicine, Faculty Hospital and Medical Faculty, Hradec Kralove, Czech Republic; J. Seibold, University of Michigan Scleroderma Program, Ann Arbor, MI, USA; S. Zeni, Dipartimento e Cattedra di Reumatologia, Università degli Studi di Milano, Milano, Italy; P. Nash, Rheumatology Research Unit, Queensland, Australia; L. Mouthon, Department of Internal Medicine, Hôpital Cochin, Paris, France; F. De Keyser, Department of Rheumatology, University of Ghent, Ghent, Belgium; M.T. Duruöz, Celal Bayar University Medical School, PM\&R Department, Manisa, Turkey; F.P. Cantatore, U.O. Reumatologia - Università degli Studi di Foggia, Foggia, Italy; G. Strauss, Department of Dermatology, University Hospital of Copenhagen, Copenhagen, Denmark; C.A. von Mülhen, Rheuma Clinic, Porto Alegre, Brasil; M.R. Pozzi, Dipartimento di Medicina, DH Reumatologia e Malattie Autoimmuni, Monza, Italy; K. Eyerich, Department of Dermatology and Allergy, TU Munich, Munich, Germany; J. Szechinski, Department of Rheumatology and Internal Diseases, Wroclaw University of Medicine, Wroclaw, Poland; M. Keiserman, Pontifical Catholic University, Porto Alegre, Brasil; F.A. Houssiau, Université catholique de Louvain, Cliniques Universitaires St-Luc, Bruxelles, Belgium; J.A.
Román-Ivorra, Hospital Universitario Dr Peset, Valencia, Spain; B. Krummel-Lorenz, Endokrinologikum, Frankfurt, Germany; M. Aringer, Department of Rheumatology, University Medical Center Carl Gustav Carus, Technical University of Dresden, Dresden, Germany; R. Westhovens, Catholic University of Leuven, Leuven, Belgium; F. Bellisai, Sezione Reumatologia - Dipartimento Medicina Clinica e Scienze Immunologiche, Università di Siena, Siena, Italy; M. Mayer, Division of Clinical Immunology and Rheumatology, University Hospital Centre Zagreb, Zagreb, Croatia; F. Stoeckl, Klinikum Darmstadt, Medizinische, Klinik III, Nephrology, Hypertension and Rheumatology, Darmstadt, Germany; M. Üprus, Department of Rheumatology, East-Tallinn Central Hospital, Tallinn, Estonia; A. Volpe, Unità di Reumatologia, Dipartimento di Medicina Generale, Ospedale Sacro Cuore - Don Calabria, Negrar, Italy; M. Buslau, Reha Rheinfelden, Rheinfelden, Switzerland; S. Yavuz, Department of Rheumatology, University of Marmara, Istanbul, Turkey; B. Granel, Service de Médecine Interne, Hôpital Nord, Marseille, France; A. Valderílio Feijó, Hospital de Clínicas da Universidade Federal do Paraná, Curitiba-Paraná, Brasil; F. Del Galdo, Scleroderma Center, Thomas Jefferson University, Philadelphia, PA, USA; S. Popa, Department of Rheumatology, Republican Clinicla Hospital, Chisinau, Republic of Moldova; T. Zenone, Department of Medicine, Unit of Internal Medicine, Valence, France; X. Ricardo Machado, Hospital de Clínicas de Porto Alegre, Porto Alegre, Brasil; M. Pileckyte, Department of Rheumatology, Kaunas University of Medicine Hospital, Kaunas, Lithuania; S. Stebbings, Dunedin School of Medicine, Dunedin, New Zealand; A. Mathieu, Rheumatology, University of Cagliari, Monserrato, Italy; A. Tulli, Clinica Dermatologica Università di Chieti, Chiety, Italy; T. Tourinho, Complexo Hospitalar Santa Casa de Porto Alegre, Porto Alegre, Brasil; R. Souza, Rheumatology Division, University of São Paulo - Faculdade de Medicina da Universidade de São Paulo, São Paulo, Brasil; R. Acayaba de Toledo, Faculdade de Medicina de São José do Rio Preto-SP, São Paulo, Brasil; L. Stamp, Department of Medicine, University of Otago, Christchurch, New Zealand; K. Solanki, Rheumatology Unit, Waikato University Hospital, Hamilton City, New Zealand; D. Veale, Department of Rheumatology, Bone and Joint Unit, St. Vincent's University Hospital, Dublin, Ireland; J. Francisco Marques Neto, Universidade Estadual de Campinas, Disciplina de Reumatologia, Departamento de Clínica Médica, Faculdade de Ciências Médicas, Campinas, Brasil; G.F. Bagnato, U.O. di Reumatologia, Policlinico Universitario "G. Martino," Messina, Italy; E. Loyo, Reumatologia e Inmunologia Clinica, Hospital Regional Universitario Jose Ma Cabral y Baez, Clinica Corominas, Santiago, Dominican Republic; S. Toloza, Hospital San Juan Batista, Catamarca, Argentina; M. Li, Department of Rheumatology, Peking Union Medical College Hospital (West Campus), Chinese Academy of Medical Sciences, Beijing, China; W. Ahmed Abdel Atty Mohamed, Unit of Rheumatology, Alexandria University, Alexandria, Egypt; V. Cobankara, Department of Internal Medicine, Division of Rheumatology, Pamukkale University, Denizli, Turkey; J. Olas, Malopolskie Centrum Reumatologii, Immunologii i Rehabilitacji, Cracow, Poland; F. Salsano, Centro per la Sclerosi Sistemica, Dipartimento di Medicina Interna, Cattedra e UOC Immunologia Clinica B, Università La Sapienza, Policlinico Umberto I, Roma, Italy; F. Oksel, Faculty of Medicine, Department of Internal Medicine, Division of Rheumatology, Ege University, Izmir, Turkey; C.M. Tanaseanu, Clinical Emergency Hospital St. Pantelimon, Bucharest, Romania; R. Foti, Servizio di Reumatologia, Clinica Medica "L. Condorelli," Catania, Italy; C. Ancuta, Rheumatology Department, Rehabilitation Hospital, Iasi, Romania; M. Vonk, Reumatology, Radboud University Nijmegen Medical Centre, Nijmegen, The Netherlands; P. Caramashi, Dipartimento di Medicina Clinica e Sperimentale, Università di Verona, Verona, Italy; L. Beretta, IRCCS Fondazione Policlinico - Mangiagalli-Regina Elena and University of Milan, Milan, Italy; A. Balbir, B. Shine Department of Rheumatology, Rambam Health Care Campus, Haifa, Israel; A. Chiàla, DIMIMP, Sezione di Reumatologia, Università degli Studi di Bari, Bari, Itália; K. Pasalic Simic, Institute of Rheumatology, Belgrade, Serbia; M. Ghio, Department of Internal Medicine, University and San Martino 
Hospital of Genoa, Genoa, Italy; B. Stamenkovic, Department of Rheumatology, Institute for Prevention and Treatment of Rheumatic and Cardiovascular Disease Ni ka Banja, Medical Faculty, University of Ni, Ni, Serbia; S. Rednic, Department of Rheumatology, "Iuliu Hatieganu" University of Medicine and Pharmacy, Cluj-Napoca, Romania; N. Host, Department of Cardiology Y, Bispebjerg University Hospital, Copenhagen, Denmark; R. Pellerito, Ospedale Mauriziano Umberto I, Torino, Italy; E. Hachulla, Service de Médecine Interne, Lille, France; D.E. Furst, Division of Rheumatology, Department of Medicine, University of California at Los Angeles, Los Angeles, CA, USA.

\section{REFERENCES}

1. Allanore Y, Avouac J, Wipff J, Kahan A. New therapeutic strategies in the management of systemic sclerosis. Expert Opin Pharmacother 2007;8:607-15.

2. Richards HL, Herrick AL, Griffin K, Gwilliam PD, Loukes J, Fortune DG. Systemic sclerosis: patients' perceptions of their condition. Arthritis Rheum 2003;49:689-96.

3. Sandqvist G, Eklund M, Akesson A, Nordenskiold U. Daily activities and hand function in women with scleroderma. Scand J Rheumatol 2004;33:102-7.

4. Baron M, Lee P, Keystone EC. The articular manifestations of progressive systemic sclerosis (scleroderma). Ann Rheum Dis 1982;41:147-52.

5. Mau W, Listing J, Huscher D, Zeidler H, Zink A. Employment across chronic inflammatory rheumatic diseases and comparison with the general population. J Rheumatol 2005;32:721-8.

6. Brower LM, Poole JL. Reliability and validity of the Duruoz Hand Index in persons with systemic sclerosis (scleroderma). Arthritis Rheum 2004;51:805-9.

7. Poole JL, Gallegos M, O'Linc S. Reliability and validity of the Arthritis Hand Function Test in adults with systemic sclerosis (scleroderma). Arthritis Care Res 2000;13:69-73.

8. Poole JL, Steen VD. The use of the Health Assessment Questionnaire (HAQ) to determine physical disability in systemic sclerosis. Arthritis Care Res 1991;4:27-31.

9. Avouac J, Guerini H, Wipff J, Assous N, Chevrot A, Kahan A, et al. Radiological hand involvement in systemic sclerosis. Ann Rheum Dis 2006;65:1088-92.

10. La Montagna G, Sodano A, Capurro V, Malesci D, Valentini G. The arthropathy of systemic sclerosis: a 12 month prospective clinical and imaging study. Skeletal Radiol 2005;34:35-41.

11. Brun B, Serup J, Hagdrup H. Radiological changes of the hands in systemic sclerosis. Acta Derm Venereol 1983;63:349-52.

12. Lovell CR, Jayson MI. Joint involvement in systemic sclerosis. Scand J Rheumatol 1979;8:154-60.

13. Cuomo G, Zappia M, Abignano G, Iudici M, Rotondo A, Valentini G. Ultrasonographic features of the hand and wrist in systemic sclerosis. Rheumatology 2009;48:1414-7.

14. Low AH, Lax M, Johnson SR, Lee P. Magnetic resonance imaging of the hand in systemic sclerosis. J Rheumatol 2009;36:961-4.

15. Allanore Y, Seror R, Chevrot A, Kahan A, Drape JL. Hand vascular involvement assessed by magnetic resonance angiography in systemic sclerosis. Arthritis Rheum 2007;56:2747-54.

16. Schumacher HR Jr. Joint and periarticular involvement in systemic sclerosis. Clin Dermatol 1994;12:277-82.

17. Tuffanelli DL, Winkelmann RK. Systemic scleroderma. A clinical study of 727 cases. Arch Dermatol 1961;84:359-71.

18. Walker UA, Tyndall A, Czirjak L, Denton C, Farge-Bancel D, Kowal-Bielecka $\mathrm{O}$, et al. Clinical risk assessment of organ manifestations in systemic sclerosis: a report from the EULAR Scleroderma Trials And Research group database. Ann Rheum Dis 2007;66:754-63.

19. Allanore Y, Meune C, Vonk MC, Airò P, Hachulla E, Caramaschi P, et al. Prevalence and factors associated with left ventricular dysfunction in the EULAR Scleroderma Trial and Research group (EUSTAR) database of patients with systemic sclerosis. Ann Rheum Dis 2010;69:218-21.

20. Tyndall A, Mueller-Ladner U, Matucci-Cerinic M. Systemic sclerosis in Europe: first report from the EULAR Scleroderma Trials And Research (EUSTAR) group database. Ann Rheum Dis 2005;64:1107.

21. LeRoy EC, Black C, Fleischmajer R, Jablonska S, Krieg T, Medsger TA Jr, et al. Scleroderma (systemic sclerosis): classification, subsets and pathogenesis. J Rheumatol 1988;15:202-5.

22. Blocka KL, Bassett LW, Furst DE, Clements PJ, Paulus HE. The arthropathy of advanced progressive systemic sclerosis. A radiographic survey. Arthritis Rheum 1981;24:874-84.

23. Walker UA, Tyndall A, Czirjak L, Denton CP, Farge-Bancel D, Kowal-Bielecka O, et al. Geographical variation of disease manifestations in systemic sclerosis: a report from the EULAR Scleroderma Trials and Research (EUSTAR) group database. Ann Rheum Dis 2009;68:856-62.

24. Resnick D. Scleroderma (progressive systemic sclerosis). In: Diagnosis of bone and joint disorders. 2nd ed. Philadelphia: W.B. Saunders Company; 1988:1191-216.

25. Cohen MJ, Persellin RH. Coexistence of rheumatoid arthritis and systemic sclerosis in four patients. Scand J Rheumatol 1982;11:241-5.

26. Armstrong RD, Gibson T. Scleroderma and erosive polyarthritis: a disease entity? Ann Rheum Dis 1982;41:141-6.

27. Baron M, Srolovitz H, Lander P, Kapusta M. The coexistence of rheumatoid arthritis and scleroderma: a case report and review of the literature. J Rheumatol 1982;9:947-50.

28. Avouac J, Airò P, Dieude P, Caramaschi P, Tiev K, Diot E, et al. Associated autoimmune diseases in systemic sclerosis define a subset of patients with milder disease: results from 2 large cohorts of European Caucasian patients. J Rheumatol 2010;37:608-14.

29. Jinnin M, Ihn H, Yamane K, Asano Y, Yazawa N, Tamaki K. Clinical features of patients with systemic sclerosis accompanied by rheumatoid arthritis. Clin Exp Rheumatol 2003;21:91-4.

30. Szucs G, Szekanecz Z, Zilahi E, Kapitany A, Barath S, Szamosi S, et al. Systemic sclerosis-rheumatoid arthritis overlap syndrome: a unique combination of features suggests a distinct genetic, serological and clinical entity. Rheumatology 2007;46:989-93.

31. Avouac J, Gossec L, Dougados M. Diagnostic and predictive value of anti-cyclic citrullinated protein antibodies in rheumatoid arthritis: a systematic literature review. Ann Rheum Dis 2006;65:845-51.

32. Morita Y, Muro Y, Sugiura K, Tomita Y. Anti-cyclic citrullinated peptide antibody in systemic sclerosis. Clin Exp Rheumatol 2008;26:542-7.

33. Marrone M, Chiala A, Tampoia M, Iannone F, Raho L, Covelli M, et al. [Prevalence of anti-CCP antibodies in systemic sclerosis]. Reumatismo 2007;59:20-4.

34. Schumacher HR Jr. Joint involvement in progressive systemic sclerosis (scleroderma): a light and electron microscopic study of synovial membrane and fluid. Am J Clin Pathol 1973;60:593-600.

35. Steen VD, Medsger TA Jr. The palpable tendon friction rub: an important physical examination finding in patients with systemic sclerosis. Arthritis Rheum 1997;40:1146-51.

36. Radic M, Martinovic Kaliterna D, Ljutic D. The level of anti-topoisomerase I antibodies highly correlates with metacarpophalangeal and proximal interphalangeal joints flexion contractures in patients with systemic sclerosis. Clin Exp Rheumatol 2006;24:407-12.

37. Valentini G, Silman AJ, Veale D. Assessment of disease activity. Clin Exp Rheumatol 2003;21:S39-41. 